\title{
HÉLÈne MAUGENDRE
} FRANÇOISE MICHEL

\section{Fibrations associées à un pinceau de courbes planes}

Annales de la faculté des sciences de Toulouse $6^{e}$ série, tome 10, $\mathrm{n}^{\mathrm{o}} 4$ (2001), p. 745-777

<http://www.numdam.org/item?id=AFST_2001_6_10_4_745_0>

C) Université Paul Sabatier, 2001, tous droits réservés.

L'accès aux archives de la revue «Annales de la faculté des sciences de Toulouse » (http://picard.ups-tlse.fr/ annales/) implique l'accord avec les conditions générales d'utilisation (http://www.numdam.org/conditions). Toute utilisation commerciale ou impression systématique est constitutive d'une infraction pénale. Toute copie ou impression de ce fichier doit contenir la présente mention de copyright.

\section{NumDam}

Article numérisé dans le cadre du programme Numérisation de documents anciens mathématiques http://www.numdam.org/ 


\title{
Fibrations associées à un pinceau de courbes planes ${ }^{(*)}$
}

\author{
Hélène Maugendre ${ }^{(1)}$, FrançOise Michel $^{(2)}$
}

\begin{abstract}
RÉSUMÉ. - Soient $f$ et $g$ deux germes de fonctions analytiques à l'origine dans $\mathbb{C}^{2}$ et $N \in \mathbb{N}^{*}$. Nous calculons explicitement l'ensemble $B$ des valeurs atypiques du pinceau $\left\{f_{a}=f+a g^{N}, a \in \mathbb{C}\right\}$. Avec notre description de $B$, nous pouvons déterminer les valeurs irrégulières à l'infini des applications polynômiales de $\mathbb{C}^{2}$ dans $\mathbb{C}$ (voir les exemples du chapitre 5). Nous comparons les fibrations de Milnor des germes $f_{a}$ du pinceau et nous exhibons un facteur commun aux polynômes caractéristiques de leurs monodromies. Lorsque $g=x$, nous montrons que la répartition de la multiplicité par dicritique est indépendante du paramètre $a$ et nous décrivons la topologie des membres génériques en fonction de la résolution minimale de $f \cdot x$.
\end{abstract}

\begin{abstract}
Let $f$ and $g$ be two holomorphic germs at the origin in $\mathbb{C}^{2}$, and let $N$ be in $\mathbb{N}^{*}$. We explicitly determine the set $B$ of atypical values of the pencil $\left\{f_{a}=f+a g^{N}, a \in \mathbb{C}\right\}$. In section 5 we use our definition of $B$ to calculate the irregular values at infinity of a polynomial map from $\mathbb{C}^{2}$ to $\mathbb{C}$. We compare the Milnor fibrations of the germs $f_{a}, a \in \mathbb{C}$. We give a common factor of the characteristic polynomials of the corresponding monodromies. When $g=x$, we describe the topology of a generic germ of the pencil in terms of the minimal resolution of $f \cdot x$.
\end{abstract}

\section{Introduction}

Notations ET DÉfinitions. - Si $g_{1}$ et $g_{2}$ sont deux germes de fonctions analytiques à l'origine de $\mathbb{C}^{2}$, la multiplicité d'intersection à l'origine entre $g_{1}$ et $g_{2}$ est notée $\left(g_{1}, g_{2}\right)_{0}$.

(*) Reçu le 26 mars 2001, accepté le 30 avril 2002

(1) Institut Fourier, Université de Grenoble I, BP 74, 38402 Saint-Martin d'Hères, courriel : Helene.Maugendre@ujf-grenoble.fr

(2) Laboratoire de mathématiques Émile Picard, Université Paul Sabatier, 118 route de Narbonne, 31062 Toulouse Cedex 4, courriel : fmichel@picard.ups-tlse.fr 
La multiplicité à l'origine de $g_{1}$ est notée $\left(g_{1}\right)_{0}$.

Les germes $g_{1}$ et $g_{2}$ sont topologiquements équivalents s'il existe un germe d'homéomorphisme $G:\left(\mathbb{C}^{2}, 0\right) \longrightarrow\left(\mathbb{C}^{2}, 0\right)$ de degré +1 tel que $g_{1} \circ$ $G=g_{2}$.

Si $g_{1}$ est un germe irréductible différent de $x$, une paramétrisation de $g_{1}$ est la donnée de $m \in \mathbb{N}^{*}$ et $\varphi(t) \in \mathbb{C}\{t\}$ tel que $\left\{g_{1}=0\right\}=\left\{\left(t^{m}, \varphi(t)\right)\right.$, $t \in \mathbb{C}\}$, de plus on choisit $m$ minimum. Le théorème de Puiseux montre l'existence d'une telle paramétrisation.

La valuation en $t$ de $g_{2}\left(t^{m}, \varphi(t)\right)$ est notée $\operatorname{val}_{t}\left(g_{2}\left(t^{m}, \varphi(t)\right)\right)$. On rappelle que, lorsque $\left(t^{m}, \varphi(t)\right)$ est une paramétrisation du germe irréductible $g_{1}$, $\operatorname{val}_{t}\left(g_{2}\left(t^{m}, \varphi(t)\right)\right)=\left(g_{1}, g_{2}\right)_{0}$.

D'autre part une composante irréductible d'un germe de courbe est appelée "branche" de la courbe.

On note $D_{\varepsilon}^{2 n}$ les boules de $\mathbb{C}^{n}$ de rayon $\varepsilon$ et $S_{\varepsilon}^{2 n-1}$ leur bord.

Soient $f$ et $g$ deux germes de fonctions analytiques à singularité, non nécessairement isolée, à l'origine de $\mathbb{C}^{2}$, tels que $f$ et $g$ n'aient aucune composante irréductible commune. Soient $N$ un entier strictement positif et $a$ un nombre complexe. Nous définissons $f_{a}:\left(\mathbb{C}^{2}, 0\right) \longrightarrow(\mathbb{C}, 0)$ par $f_{a}(x, y)=f(x, y)+a(g(x, y))^{N}$. Nous considérons le germe d'application analytique $\Phi_{a}=\left(g, f_{a}\right):\left(\mathbb{C}^{2}, 0\right) \longrightarrow\left(\mathbb{C}^{2}, 0\right)$ défini par :

$$
\Phi_{a}(x, y)=\left(g, f_{a}\right)(x, y)=\left(g(x, y), f_{a}(x, y)\right) .
$$

Le germe $\Phi_{a}$ est fini car $g$ et $f_{a}$ n'ont aucune composante commune.

Le lieu des zéros $D$ du déterminant $\hat{D}=(\partial g / \partial x)(\partial f / \partial y)-(\partial g / \partial y)(\partial f / \partial x)$ de la matrice jacobienne de $\Phi_{a}$ est le lieu critique de $\Phi_{a}$.

Le rôle de l'entier $N$, qui paraît arbitraire dans la définition de $f_{a}$, deviendra naturel dans l'étude détaillée des pinceaux de la forme $f(x, y)+a x^{N}$ (voir chapitre 4). De tels pinceaux déterminent le comportement à l'infini des applications polynômiales $P$, de $\mathbb{C}^{2}$ dans $\mathbb{C}$. L'entier $N$ est alors égal au degré du polynôme $P$.

Par convention, nous choisissons des coordonnées dans $\mathbb{C}^{2}$ pour que $\{x=0\}$ ne soit pas une composante de $D \cup f^{-1}(0)$.

ÉNONCÉ DES PRINCIPAUX RÉSULTATS. - Traditionnellement, l'ouvert d'équisingularité du pinceau $f+a g^{N}$ est le plus grand ouvert $U$ de $\mathbb{C}$ tel que, quels que soient $a, b \in U, f_{a}$ et $f_{b}$ ont même type topologique. 
Ici nous construisons explicitement un ensemble fini $B$, de nombres complexes, de la façon suivante.

Considérons l'ensemble (éventuellement vide) des branches $\gamma_{j}$ de $D, 1 \leqslant$ $j \leqslant k$ paramétrées par $\left(t^{m_{j}}, \varphi_{j}(t)\right)$ telles que $\operatorname{val}_{t} f\left(\left(t^{m_{j}}, \varphi_{j}(t)\right)\right)=N\left(g, \gamma_{j}\right)_{0}$. Nous obtenons :

$$
\begin{aligned}
& f\left(\left(t^{m_{j}}, \varphi_{j}(t)\right)\right)=b_{j} t^{N\left(g, \gamma_{j}\right)_{0}}+\sum_{i \geqslant N\left(g, \gamma_{j}\right)_{0}+1} c_{i} t^{i} \\
& g\left(\left(t^{m_{j}}, \varphi_{j}(t)\right)\right)=d_{j} t^{\left(g, \gamma_{j}\right)_{0}}+\sum_{i \geqslant\left(g, \gamma_{j}\right)_{0}+1} d_{i}^{\prime} t^{i} .
\end{aligned}
$$

Posons $B_{1}=\left\{-b_{j} d_{j}^{-N}, 1 \leqslant j \leqslant k\right\}$.

Si $f$ est non réduite ou s'il existe au moins une branche $\gamma$ de $D$ paramétrée par $\left(t^{m}, \varphi(t)\right)$ telle que val $t f\left(\left(t^{m}, \varphi(t)\right)\right)>N(g, \gamma)_{0}$, alors $B=B_{1} \cup\{0\}$, sinon $B=B_{1}$.

Dans le chapitre 2 nous démontrons :

THÉORÈme 1. - L'ouvert $\mathbb{C} \backslash B$, est l'ouvert $U$ d'équisingularité du pinceau $f+a g^{N}$.

L'existence d'un tel ouvert est connue depuis O. Zariski (voir [Z] mais aussi [T1]). Dans [L-W], D.T. Lê et C. Weber donnent une caractérisation de l'ensemble fini $\mathbb{C} \backslash U$ en fonction de la résolution minimale du pinceau.

Le théorème 1 présente l'avantage de déterminer $B$ algorithmiquement à partir de $f$ et $g$ sans référence au paramètre $a$. Il fournit aussi la caractérisation implicite suivante de $U$ : "le paramètre $a$ appartient à $U$ si et seulement si la multiplicité d'intersection de $f_{a}$ avec chaque branche de $D$ est minimale."

Ensuite nous comparons les fibrations de Milnor des membres du pinceau $f+a g^{N}$ et nous déterminons un facteur commun aux polynômes caractéristiques des monodromies associées.

Plus précisément, pour $a \in \mathbb{C}^{*}$ et $\varepsilon$ suffisamment petit, nous considérons la décomposition minimale de Waldhausen $\bigcup_{\text {finie }} V_{S}$ de $S_{\varepsilon}^{3}$ admettant les composantes de l'entrelacs de $f f_{a} g$ pour feuilles de Seifert. À chaque variété de Seifert $V_{S}$ nous associons l'invariant topologique $q(S)$ (voir définition 5 paragraphe 3.1). 
ThÉoRÈme 2. - Pour tout a $\in \mathbb{C}^{*}$, si $N>\min q(S)$, la variété de Waldhausen $W^{N}=\bigcup_{q(S)<N} V_{S}$ satisfait les propriétés suivantes :

1. $\varphi_{a}=f_{a} /\left|f_{a}\right|_{\mid}: W^{N} \longrightarrow S^{1}$ et $\varphi=f /|f|_{\mid}: W^{N} \longrightarrow S^{1}$ sont des fibrations isomorphes;

2. si $F_{a}^{N}$ est une fibre de $\varphi_{a}$, alors $\chi\left(F_{a}^{N}\right)=\left(g, f_{a}\right)_{\circ}-\left(f_{a}, \Gamma^{N}\right)_{\circ}$ ò̀ $\chi\left(F_{a}^{N}\right)$ est la caractéristique d'Euler de $F_{a}^{N}$, et $\Gamma^{N}$ est le produit des composantes $\gamma$ de $D$ telles que $(f, \gamma)_{\circ} /(g, \gamma)_{\circ}<N$;

3. la résolution minimale de $f \cdot g$ définit un ensemble fini $I_{N}$ et des entiers positifs $r_{N}, v_{i}\left(f_{a}\right)$, et val $_{E_{i}}\left(f_{a}\right)$ où $i \in I_{N}$, tels que le polynôme $\Lambda_{a}^{N}(t)$ défini par :

$$
\Lambda_{a}^{N}(t)=\left(t^{r_{N}}-1\right) \prod_{i \in I_{N}}\left(t^{v a l_{E_{i}}\left(f_{a}\right)}-1\right)^{v_{i}\left(f_{a}\right)-2}
$$

soit le polynôme caractéristique de la monodromie de $f_{a}^{N}$. De plus, $\Lambda_{a}^{N}$ divise le polynôme caractéristique de la monodromie de $f$ et celui de la monodromie de $f_{a}$.

La variété $W^{N}$ est construite (3.2 et 3.3) à l'aide du carrousel de Lê (voir [L1]). Le théorème 3 (3.3) montre que $\varphi_{a}$ est une "sous-fibration de Milnor" commune à tous les membres du pinceau et entraîne les points 1 et 2 du théorème 2 . Le point 3 du théorème 2 est démontré au paragraphe 4.1 (proposition 6).

En toutes dimensions, C. Caubel ([C1], [C2] et [C3]) étudie les fibrations de Milnor des pinceaux de germes d'hypersurfaces et retrouve, en particulier, les résultats de I.N. Iomdine, D.T. Lê, D. Siersma, M. Tibar (voir [I], [L2], [S], [Ti]) qui concernaient les pinceaux de la forme $f\left(z_{0}, \ldots, z_{n}\right)+a z_{0}^{N}$ avec $N$ "grand". Pour d'autres familles voir aussi [Sc].

Lorsque l'on considère les valeurs atypiques à l'infini des applications polynômiales de $\mathbb{C}^{2}$ dans $\mathbb{C}$, on est amené à regarder des pinceaux de la forme $f(x, y)+a x^{N}$. C'est pourquoi, au chapitre 4, nous faisons une étude détaillée de ce pinceau au moyen de la résolution minimale de $f \cdot x$. Les résultats de ce chapitre 4 se généralisent directement aux pinceaux $f+a g^{N}$ lorsque $g$ est irréductible. Au chapitre 5 (exemple 3) nous donnons des exemples de détermination des valeurs atypiques à l'infini d'une application polynômiale de $\mathbb{C}^{2}$ dans $\mathbb{C}$.

Pour les pinceaux $f(x, y)+a x^{N},\{q(S)\}$ est l'ensemble des quotients polaires de $f$ pour la direction $x$ (voir [LMW1]). En 4.2 et 4.3, nous supposons 
$N>\min \{q(S)\}$. Pour tout $a \in \mathbb{C}^{*}$, nous considérons la résolution minimale $\pi$ du germe produit $f \cdot f_{a} \cdot x$. Nous notons $\mathcal{A}\left(f \cdot f_{a} \cdot x\right)$ l'arbre orienté qui représente $\pi$. Étant donné un sommet dicritique $S$ de $\mathcal{A}\left(f \cdot f_{a} \cdot x\right)$ (voir le corollaire 7 du paragraphe 4.2), nous notons $\varepsilon$ l'arête qui arrive en $S$. Pour tout $b \in \mathbb{C}, f_{b, \varepsilon}$ désigne le produit (avec multiplicité) des composantes de $f_{b}$ dont la géodésique passe par $\varepsilon$ (voir le paragraphe 4.3). Nous montrons (proposition 9) :

$$
\left(f_{b, \varepsilon}, x\right)_{0}=\left(f_{a, \varepsilon}, x\right)_{0}, \text { quel que soit } b \in \mathbb{C} .
$$

Cette égalité est non trivale si $b$ appartient à $B$. Elle montre que la "répartition de la multiplicité" par dicritique est indépendante de la valeur du paramètre $a$ de la déformation $f(x, y)+a x^{N}$. Ceci limite les variations de la topologie des fibres spéciales et s'applique en particulier aux singularités à l'infini des applications polynômiales de $\mathbb{C}^{2}$ dans $\mathbb{C}$.

Ensuite, pour tout $a \in U$, nous déterminons, dans les théorèmes 4 et 5 du paragraphe 4.4, l'arbre de la résolution minimale de $f_{a} \cdot x$ et de $f \cdot f_{a} \cdot x$ à partir de celui de $f \cdot x$.

Les pinceaux $f(x, y)+a x^{N}$ lorsque $N \leqslant \min \{q(S)\}$ sont dits dégénérés. Ils sont étudiés au paragraphe 4.5 .

\section{Caractérisation des valeurs atypiques}

Considérons l'ensemble fini $B$ et l'ouvert d'équisingularité $U=\mathbb{C} \backslash B$ définis dans l'introduction.

DÉFINITION 1. - Si $a \in U$, a est une valeur régulière du pinceau et $f_{a}$ est un germe générique du pinceau.

Si $b \in B, b$ est une valeur atypique du pinceau et $f_{b}$ est un germe spécial du pinceau.

DÉFINITION 2. - La courbe jacobienne de $\left(g, f_{a}\right)$ est l'adhérence du lieu critique de $\Phi_{a}$ auquel on enlève les éventuelles branches de $\left(f_{a} \cdot g\right)^{-1}(0)$.

On obtient donc, pour tout $a \in \mathbb{C}, \Gamma_{a}=\overline{D \backslash\left(\left\{f_{a} \cdot g=0\right\} \cap D\right)}$.

La courbe discriminante $\Delta_{a}$ est l'image de $\Gamma_{a}$ par l'application $\Phi_{a}$.

Remarque 1. - Pour $a \in U, f_{a}$ est à singularité isolée à l'origine et $\Gamma_{a}$ est alors constituée de la réunion de la courbe jacobienne $\Gamma_{0}$ de $(g, f)$ et des éventuelles composantes non réduites de $f^{-1}(0)$. 
Soient $(u, v)$ les coordonnées complexes de $\Phi_{a}\left(\left(\mathbb{C}^{2}, 0\right)\right)$ et $\delta$ une branche de $\Delta_{a}$. Alors il existe un nombre rationnel $q_{\delta} / p_{\delta}$ strictement positif $\left(\operatorname{pgcd}\left(q_{\delta}, p_{\delta}\right)=1\right)$, un entier $m>0$ tels qu'un développement de Puiseux de $\delta$ soit donné par :

$$
u=v^{q_{\delta} / p_{\delta}}\left(\alpha+\sum_{k \in \mathbb{N}^{*}} \beta_{k} v^{k / m}\right)
$$

avec $\alpha \in \mathbb{C}^{*}\left(\operatorname{car}\{u=0\}\right.$ n'est pas une branche de $\left.\Delta_{a}\right)$ et $\beta_{k} \in \mathbb{C}$.

DÉFInITION 3. - L'ensemble des quotients jacobiens de $\left(g, f_{a}\right)$ est l'ensemble $Q_{a}$ des nombres rationnels $p_{\delta} / q_{\delta}$ associés aux branches $\delta$ de $\Delta_{a}$.

Si g est une forme linéaire, $Q_{a}$ est l'ensemble des quotients polaires de $f_{a}$ pour la direction $g$.

Cet ensemble est un invariant du type topologique de la paire de germes $\left(g, f_{a}\right)$. Lorsque $g$ est une forme linéaire transverse à $f$ voir [L-M$\mathrm{W} 1]$, sinon voir [Ma2]. La proposition suivante qui nous sera utile pour les démonstrations se vérifie facilement (voir [L-M-W1] ou [Ma2]) :

Proposition a. - L'ensemble $Q_{a}$ est égal à l'ensemble constitué des nombres rationnels $q_{\gamma}\left(f_{a}\right)=\left(f_{a}, \gamma\right)_{0} /(g, \gamma)_{0}$, où $\gamma$ est une branche de $\Gamma_{a}$.

REMARQUE 2. - Si une branche $\gamma$ de $\Gamma_{a}(a \neq 0)$ n'est pas une branche de $\Gamma_{0}$, alors $\gamma$ est une branche de $f^{-1}(0)$ et correspond à un quotient jacobien égal à $N$ pour $\left(g, f_{a}\right)$.

Proposition 1. - Si $p / q$ appartient à $Q_{0}$ et $p / q<N$ alors $p / q$ appartient à $Q_{a}$ quel que soit a dans $\mathbb{C}$. De plus, si $a \in U$, alors $\max Q_{a} \leqslant N$.

Démonstration. - Comme $p / q<N$, toute branche $\gamma$ de $\Gamma_{0}$ de quotient $p / q$ est une branche de $\Gamma_{a}$. On doit vérifier que le quotient est le même pour $\Gamma_{0}$ et $\Gamma_{a}$.

On a $f_{a}(x, y)=f(x, y)+a(g(x, y))^{N}$. Si $\left(t^{m}, \varphi(t)\right)$ est une paramétrisation de $\gamma$, on a $\left(f_{a}, \gamma\right)_{0} /(g, \gamma)_{0}=\operatorname{val}_{t}\left(f_{a}\left(t^{m}, \varphi(t)\right)\right) / \operatorname{val}_{t}\left(g\left(t^{m}, \varphi(t)\right)\right)$ et $\left(f_{a}, \gamma\right)_{0} /(g, \gamma)_{0}=\operatorname{val}_{t}\left(f\left(t^{m}, \varphi(t)\right)+a\left(g\left(t^{m}, \varphi(t)\right)\right)^{N}\right) / \operatorname{val}_{t}\left(g\left(t^{m}, \varphi(t)\right)\right)$.

Si $\operatorname{val}_{t}\left(f\left(t^{m}, \varphi(t)\right)\right) / \operatorname{val}_{t}\left(g\left(t^{m}, \varphi(t)\right)\right)<N$, c'est-à-dire si le quotient jacobien de $f$ associé à $\gamma$ est strictement plus petit que $N$, alors le quotient jacobien pour $f_{a}$ associé à $\gamma$ est le mème que celui de $f$.

Un calcul analogue montre que si $a \in U$ alors $\max Q_{a} \leqslant N$. 
Fibrations associées à un pinceau de courbes planes

Nous appelons $Q_{a}^{N}$ le sous-ensemble de $Q_{a}$ défini comme suit :

$$
Q_{a}^{N}=\left\{p_{\delta} / q_{\delta} \in Q_{a} \text { tels que } p_{\delta} / q_{\delta}<N\right\}
$$

Nous notons $\Delta_{a}^{N}$ l'ensemble des branches de $\Delta_{a}$ dont le quotient jacobien associé appartient à $Q_{a}^{N}$. La proposition 1 implique :

Corollaire 1. - Si $N$ est strictement supérieur au plus petit quotient jacobien de $(g, f)$, alors, pour tout $a \in \mathbb{C}$, l'ensemble $Q_{a}^{N}$ n'est pas vide, de plus, $Q_{a}^{N}=Q_{0}^{N}$.

On va relier l'ensemble des valeurs atypiques $B$, défini dans l'introduction, à la courbe jacobienne et aux quotients jacobiens de $(g, f)$.

Remarque 3. - 1. Les définitions de $B$ et de $Q_{0}$ impliquent que si $N$ n'appartient pas à $Q_{0}$, alors, soit $B$ est vide, soit $B=\{0\}$.

2. S'il existe $b \in \mathbb{C}$ tel que $f_{b}$ soit non réduite, alors $b \in B$. En effet, si $b=0$ et si $f_{b}$ n'est pas réduite, c'est par définition; si $b \neq 0$ et si $f_{b}$ n'est pas réduite, alors $f_{b}^{-1}(0)$ et $D$ ont une branche commune paramétrée par $\left(t^{m}, \varphi(t)\right)$, et $f_{b}\left(t^{m}, \varphi(t)\right)=0$, donc $f\left(t^{m}, \varphi(t)\right)+b\left(g\left(t^{m}, \varphi(t)\right)\right)^{N}=0$, ce qui implique $b \in B$.

Proposition 2. - Il existe $b \in \mathbb{C}$ tel que $\max Q_{b} \geqslant N$ si et seulement si $B \neq \emptyset$. Dans ce cas on $a Q_{a}=Q_{0}^{N} \cup\{N\}$ quel que soit $a \in U$.

Démonstration. - Supposons qu'il existe $b \in \mathbb{C}$ avec $\max Q_{b} \geqslant N$, alors il existe une branche $\gamma$ de $\Gamma_{b}$ paramétrée par $\left(t^{m}, \varphi(t)\right)$ telle que $\operatorname{val}_{t} f_{b}\left(t^{m}, \varphi(t)\right) \geqslant N(g, \gamma)_{0}$. On a aussi $\operatorname{val}_{t} f\left(t^{m}, \varphi(t)\right) \geqslant N(g, \gamma)_{0}$. On a ainsi $f_{b}\left(t^{m}, \varphi(t)\right)=c t^{n}+\sum_{i \geqslant n+1} c_{i} t^{i}, c \neq 0$.

On développe $g\left(t^{m}, \varphi(t)\right)=d t^{(g, \gamma)_{0}}+\sum_{i \geqslant(g, \gamma)_{0}+1} d_{i} t^{i}$

Deux cas sont à considérer :

1. Si $n>N(g, \gamma)_{0}$, alors $f\left(t^{m}, \varphi(t)\right)=-b d^{N} t^{N(g, \gamma)_{0}}+\ldots$ et donc $b \in B$.

2. Si $n=N(g, \gamma)_{0}$, alors $f\left(t^{m}, \varphi(t)\right)=\left(c-b d^{N}\right) t^{N(g, \gamma)_{0}}+\ldots$.

Si $c \neq b d^{N}$, alors $b-c d^{-N} \in B$.

Enfin si $c=b d^{N}$, alors $0 \in B$ car, soit on a $f\left(t^{m}, \varphi(t)\right)=0$ et $f$ est non réduite, soit $\operatorname{val}_{t}\left(f\left(t^{m}, \varphi(t)\right)\right)>N(g, \gamma)_{0}$ et $\max Q_{0}>N$; dans tous les cas $0 \in B$ par définition. 
On vient de montrer que s'il existe $b$ tel que $\max Q_{b} \geqslant N$, on a $B \neq \emptyset$.

Si $B_{1} \neq \emptyset$, alors $\max Q_{a} \geqslant N$ pour tout $a \notin B_{1}$. Dans ce cas la proposition 1 et le corollaire 1 impliquent $Q_{a}=Q_{0}^{N} \cup\{N\}$ pour tout $a \in U$.

Sinon, $B=\{0\}$, et alors, soit $f$ n'est pas réduite et donc $Q_{a}=Q_{0}^{N} \cup\{N\}$ pour tout $a \neq 0$, soit max $Q_{0}>N$ et alors il existe une branche $\gamma$ de $\Gamma_{0}$ paramétrée par $\left(t^{m}, \varphi(t)\right)$ telle que $\operatorname{val}_{t}\left(f\left(t^{m}, \varphi(t)\right)\right)>N(g, \gamma)_{0}$. Par conséquent pour tout $a \neq 0, \operatorname{val}_{t}\left(f_{a}\left(t^{m}, \varphi(t)\right)\right)=N(g, \gamma)_{0}$ et $Q_{a}=Q_{0}^{N} \cup$ $\{N\}$.

La proposition 2 implique en particulier :

Corollaire 2. - Si $B=\emptyset$ alors $Q_{a}=Q_{0}=Q_{0}^{N}$ quel que soit $a \in \mathbb{C}$.

Démonstration $d u$ théorème 1. - Comme $a \notin B, f_{a}$ est réduite. Si $f_{b}$ n'est pas réduite, alors $b \in B$ (d'après la remarque 3 ) et $f_{b}$ n'est pas topologiquement équivalent à $f_{a}$.

Maintenant il suffit donc de considérer le cas où $f_{b}$ est réduite. Si $c \in \mathbb{C}$, le germe jacobien $\hat{\Gamma}_{c}$ de $\left(g, f_{c}\right)$ est le produit des composantes de $\hat{D}$ qui ne divisent pas $g \cdot f_{c}$. Si $f_{a}$ et $f_{b}$ sont réduites on a $\hat{\Gamma}_{a}=\hat{\Gamma}_{b}=\prod_{i=1}^{n} \hat{\gamma}_{i}^{k_{i}}$, où les germes irréductibles $\hat{\gamma}_{i}$ et $\hat{\gamma}_{j}$ sont distincts pour $i \neq j$.

Dans [Ma2], on montre que pour tout $c \in \mathbb{C}$, il existe $\varepsilon, \theta, \eta, 0<\eta \ll$ $\theta \ll \varepsilon$ suffisamment petits tels que la restriction $f_{c \mid}$ de $f_{c}$ à $X_{c}=f_{c}^{-1}\left(S_{\eta}^{1}\right) \cap$ $g^{-1}\left(D_{\theta}^{2}\right) \cap D_{\varepsilon}^{4}$ soit (à isotopie près) la fibration de Milnor de $f_{c}$. C'est une version particulière de la fibration de Milnor construite à partir du carrousel de Lê. On note $F_{c}$ une fibre de $f_{c \mid}$. Comme dans [L1] on a :

$$
\begin{aligned}
& \chi\left(F_{a}\right)=\left(g, f_{a}\right)_{0}-\left(\hat{\Gamma}_{a}, f_{a}\right)_{0}, \\
& \chi\left(F_{b}\right)=\left(g, f_{b}\right)_{0}-\left(\hat{\Gamma}_{b}, f_{b}\right)_{0} .
\end{aligned}
$$

Comme $f_{a}(x, y)=f_{b}(x, y)+(a-b)(g(x, y))^{N}$, on a $\left(g, f_{a}\right)_{0}=\left(g, f_{b}\right)_{0}$ quel que soit $b \in \mathbb{C}$.

D'autre part,

$$
\left(\hat{\Gamma}_{a}, f_{a}\right)_{0}=\sum_{i=1}^{n} k_{i}\left(\hat{\gamma}_{i}, f_{a}\right)_{0} \text { et }\left(\hat{\Gamma}_{b}, f_{b}\right)_{0}=\left(\hat{\Gamma}_{a}, f_{b}\right)_{0}=\sum_{i=1}^{n} k_{i}\left(\hat{\gamma}_{i}, f_{b}\right)_{0}
$$


Soit $\left(t^{m_{i}}, \varphi_{i}(t)\right)$ une paramétrisation de $\hat{\gamma}_{i}$. On a :

$$
(*)\left\{\begin{aligned}
q_{\gamma_{i}}\left(f_{a}\right) & =\frac{\operatorname{val}_{t}\left(f_{a}\left(t^{m_{i}}, \varphi_{i}(t)\right)\right)}{\left(g, \hat{\gamma}_{i}\right)_{0}} \\
q_{\gamma_{i}}\left(f_{b}\right) & =\frac{\operatorname{val}_{t}\left(f_{a}\left(t^{m_{i}}, \varphi_{i}(t)\right)+(b-a) g\left(t^{m_{i}}, \varphi(t)\right)^{N}\right)}{\left(g, \hat{\gamma}_{i}\right)_{0}} .
\end{aligned}\right.
$$

Comme $a \in U, \max Q_{a} \leqslant N$ (voir la proposition 1). Si $q_{\gamma_{i}}\left(f_{b}\right) \leqslant N$, par $(*)$, on a $\left(\hat{\gamma}_{i}, f_{a}\right)_{0}=\left(\hat{\gamma}_{i}, f_{b}\right)_{0}$.

Par conséquent :

1. si $b \notin B, \max Q_{b} \leqslant N$ et $\left(\hat{\Gamma}_{a}, f_{a}\right)_{0}=\left(\hat{\Gamma}_{b}, f_{b}\right)_{0}$. De plus, $f_{a}$ et $f_{b}$ sont à singularité isolée et en particulier $F_{a}$ et $F_{b}$ sont connexes. Donc $\chi\left(F_{a}\right)=\chi\left(F_{b}\right)$ implique $\operatorname{rang}_{\mathbb{Z}} H_{1}\left(F_{a}, \mathbb{Z}\right)=\operatorname{rang}_{\mathbb{Z}} H_{1}\left(F_{b}, \mathbb{Z}\right)$. Le théorème de $\mu$-constant pour les germes de courbes ([L1]) implique alors que $f_{a}$ et $f_{b}$ sont topologiquement équivalentes ;

2. si $b \in B$ et $f_{b}$ est réduite, il existe des composantes irréductibles $\hat{\gamma}_{i_{1}}, \ldots, \hat{\gamma}_{i_{s}}$ de $\hat{\Gamma}_{a}=\hat{\Gamma}_{b}$ telles que $q_{\hat{\gamma}_{i_{j}}}\left(f_{b}\right)>N=q_{\hat{\gamma}_{i_{j}}}\left(f_{a}\right)$. Alors $\left(\hat{\gamma}_{i}, f_{a}\right)_{0}=\left(\hat{\gamma}_{i}, f_{b}\right)_{0}$ si $i \notin\left\{i_{j}, j=1, \ldots, s\right\}$ et $\left(\hat{\gamma}_{i}, f_{a}\right)_{0}=N\left(g, \hat{\gamma}_{i}\right)_{0}<$ $\left(\hat{\gamma}_{i}, f_{b}\right)_{0}$ si $i \in\left\{i_{j}, j=1, \ldots, s\right\}$. Donc $\chi\left(F_{b}\right)<\chi\left(F_{a}\right)$, ce qui implique que $f_{a}$ et $f_{b}$ ne sont pas topologiquement équivalentes.

Commentaires. - On peut montrer topologiquement, sans utiliser le théorème $\mu$-constant, que si $b \notin B$ alors $f_{a}$ et $f_{b}$ sont topologiquement équivalentes. Au paragraphe 3.2, la construction de la décomposition minimale de Waldhausen pour $f_{a}$ et $f_{b}$ donne, entre autre, une telle preuve topologique directe.

\section{Fibration commune aux germes du pinceau $f(x, y)+a(g(x, y))^{N}$}

\subsection{Graphe coloré de la décomposition minimale de Waldhausen de $S_{\varepsilon}^{3}$ pour $f \cdot f_{a} \cdot g$}

Soit $\ell$ un germe de fonction analytique à l'origine de $\mathbb{C}^{2}$.

DÉfINITION 4. - Une décomposition de Waldhausen de $S_{\varepsilon}^{3}$ pour $\ell$ est une décomposition de $S_{\varepsilon}^{3}$ en une réunion finie de variétés de Seifert (variétés connexes qui sont munies d'un feuilletage orienté en cercles) telle que les composantes de l'entrelacs orienté et pondéré $K_{\ell}=\ell^{-1}(0) \cap S_{\varepsilon}^{3}$ soient des feuilles de certaines de ces variétés. 
Remarque 4. - Deux variétés de Seifert d'une telle décomposition s'intersectent en au plus un tore.

Le graphe de Waldhausen associé à une décomposition de Waldhausen de $S_{\varepsilon}^{3}$ pour $\ell$, noté $G(\ell)$ se construit comme suit.

Chaque sommet $S$ de l'arbre correspond à une variété de Seifert $V_{S}$ de la décomposition de Waldhausen de $S_{\varepsilon}^{3}$ pour $\ell$ considérée. Deux sommets sont reliés par une arête si les variétés de Seifert qui leur correspondent s'intersectent (toujours suivant un unique tore).

RemaRQUE 5. - Comme le graphe $G(\ell)$ représente une décomposition de Waldhausen de $S_{\varepsilon}^{3}, G(\ell)$ est un arbre.

Soit $a \in \mathbb{C} \backslash\{0\}$. Pour la construction de $G\left(f \cdot f_{a} \cdot g\right)$, on met autant de flèches rouges (respectivement bleues, respectivement noires) à un sommet $S$ qu'il existe de feuilles de la variété de Seifert $V_{S}$ (correspondant à ce sommet) qui sont des composantes de $K_{f_{a}}$ (respectivement $K_{f}$, respectivement $K_{g}$ ).

DÉFINITION 5. - Si S est un sommet de $G\left(f \cdot f_{a} \cdot g\right)$, le quotient topologique de $S$ pour $f_{a}$ (respectivement $f$ ), noté $q_{a}(S)$ (respectivement $q(S)$ ) est égal à $\mathcal{L}\left(K_{f_{a}}, \rho\right) / \mathcal{L}\left(K_{g}, \rho\right)$, (respectivement $\left.\mathcal{L}\left(K_{f}, \rho\right) / \mathcal{L}\left(K_{g}, \rho\right)\right)$, où $\rho$ est une feuille de la variété de Seifert $V_{S}$ et $\mathcal{L}($,$) désigne le nombre$ d'enlacement dans $S_{\varepsilon}^{3}$.

\subsection{Obtention de la décomposition minimale de Waldhausen de $S_{\varepsilon}^{3}$ pour $f \cdot f_{a} \cdot g$ via le carrousel}

Dans ce paragraphe, $N>\min Q_{0}$ et $a \in \mathbb{C} \backslash\{0\}$. Le cas $N \leqslant \min Q_{0}$ est dégénéré et traité au paragraphe 4.5 .

D'après [Ma2], nous savons qu'il existe des réels strictement positifs $\eta, \theta, \varepsilon$ vérifiant $0<\eta \ll \theta \ll \varepsilon$, tels que pour $\Sigma_{a}=\left[\left(f_{a}^{-1}\left(S_{\eta}^{1}\right) \cap g^{-1}\left(D_{\theta}^{2}\right)\right) \cup\right.$ $\left.\left(f_{a}^{-1}\left(D_{\eta}^{2}\right) \cap g^{-1}\left(S_{\theta}^{1}\right)\right)\right] \cap D_{\varepsilon}^{4}$ on a le résultat suivant :

THÉORÈME b. - Il existe $\varepsilon, \theta, \eta$ des réels suffisamment petits, avec $0<\eta \ll \theta \ll \varepsilon$, tels qu'il existe un difféomorphisme (à coins) $\Psi$ de $\Sigma_{a}$ sur $S_{\varepsilon}^{3}$ tel que $\Psi\left(K_{f}^{\Sigma_{a}}\right)=K_{f}, \Psi\left(K_{f_{a}}^{\Sigma_{a}}\right)=K_{f_{a}}$ et $\Psi\left(K_{g}^{\Sigma_{a}}\right)=K_{g}$ ò̀ $K_{f}^{\Sigma_{a}}=f^{-1}(0) \cap \Sigma_{a}, K_{f_{a}}^{\Sigma_{a}}=f_{a}^{-1}(0) \cap \Sigma_{a}$ et $K_{g}^{\Sigma_{a}}=g^{-1}(0) \cap \Sigma_{a}$.

Par conséquent, établir la décomposition minimale de Waldhausen de $S_{\varepsilon}^{3}$ pour $f \cdot f_{a} \cdot g$ revient à isotopie près à construire la décomposition minimale de Waldhausen de $\Sigma_{a}$ pour $f \cdot f_{a} \cdot g$. 
Quitte à diminuer $\eta, \theta, \varepsilon$, nous pouvons les choisir de sorte que l'intersection de $\Delta_{a}$ avec le bord de $D_{\theta}^{2} \times D_{\eta}^{2}$ soit contenue dans $D_{\theta}^{2} \times S_{\eta}^{1}$.

Par ailleurs, soit $\Delta_{a, i}$ la réunion des composantes irréductibles de $\Delta_{a}$ qui admettent $p_{i} / q_{i}$ pour quotient jacobien, $1 \leqslant i \leqslant k$. Il est facile de constater, à l'aide des développements de Puiseux des branches de $\Delta_{a}$, que, plus le quotient jacobien augmente, plus la tresse $K_{\Delta_{a, i}}=\Delta_{a, i} \cap\left(D_{\theta}^{2} \times S_{\eta}^{1}\right)$ s'éloigne de l'âme de $\left(D_{\theta}^{2} \times S_{\eta}^{1}\right)$.

Par conséquent, il existe un réel strictement positif $\theta^{\prime}, 0<\theta^{\prime}<\theta$ tel que :

$$
\left(\Delta_{a} \cup \Phi_{a}(\{f=0\})\right) \cap\left(D_{\theta^{\prime}}^{2} \times S_{\eta}^{1}\right)=\Delta_{a}^{N} \cap\left(D_{\theta^{\prime}}^{2} \times S_{\eta}^{1}\right) .
$$

REMARQUE 6. - La courbe $\left\{a u^{N}=v\right\}$ est une branche de $\Delta_{a}$ si et seulement si $f$ est à singularité non isolée à l'origine.

On écrit

$\{N\} \cup Q_{a}=\left\{p_{i} / q_{i}, i=1, \ldots, k,\left(p_{i} / q_{i}\right)<\left(p_{i+1} / q_{i+1}\right)\right.$, p.g.c.d. $\left.\left(p_{i}, q_{i}\right)=1\right\}$.

RemaRque 7. - Il existe $m, 1 \leqslant m<k$, tel que $p_{m+1} / q_{m+1}=N$.

D'après [L-M-W1], chapitre 2, nous avons le résultat suivant :

LEMME c. - Il existe des réels $\alpha$ et $\theta_{i}, 1 \leqslant i \leqslant k$, avec $0<\alpha \ll \theta_{1}<$ $\theta_{2}<\cdots<\theta_{k}$ tels que si :

$$
\begin{gathered}
Z_{1}=\left(D_{\theta_{1}}^{2} \times \stackrel{\circ}{S_{\eta}^{1}}\right) \backslash\left(\stackrel{\circ}{D_{\alpha}^{2}} \times S_{\eta}^{1}\right) \\
Z_{i}=\left(D_{\theta_{i}}^{2} \times S_{\eta}^{1}\right) \backslash\left(D_{\theta_{i-1}}^{2} \times S_{\eta}^{1}\right), 2 \leqslant i \leqslant k,
\end{gathered}
$$

alors $K_{\Delta_{a}} \cap Z_{i}=\Delta_{a} \cap\left(D_{\theta}^{2} \times S_{\eta}^{1}\right) \cap Z_{i}=K_{\Delta_{a, i}}$, pour tout $i \neq m+1$, et $\left(K_{\Delta_{a}} \cup\left\{a u^{N}=v\right\}\right) \cap Z_{m+1}=\left(\Delta_{a} \cup \Phi_{a}(\{f=0\})\right) \cap\left(D_{\theta}^{2} \times S_{\eta}^{1}\right) \cap Z_{m+1}=$ $K_{\Delta_{a, m+1}} \cup \Phi_{a}\left(K_{f}^{\Sigma_{a}}\right)$.

Les $Z_{i}$ sont appelées les zones jacobiennes.

On peut choisir $\theta^{\prime}$ tel que $D_{\theta^{\prime}}^{2} \times S_{\eta}^{1}=\left(\bigcup_{i=1}^{m} Z_{i}\right) \cup\left(D_{\alpha}^{2} \times S_{\eta}^{1}\right)$.

\section{Construction}

Le moyen d'obtenir la décomposition minimale de Waldhausen de $\Sigma_{a}$ pour $f \cdot f_{a} \cdot g$ est le suivant. Nous savons que la restriction $\Phi_{\mid \Sigma_{a}}$ de $\Phi_{a}$ à $\Sigma_{a}$ est un revêtement ramifié qui admet $\Gamma_{a} \cap \Sigma_{a}$ et les éventuelles composantes non réduites de $K_{f_{a} g}$ pour lieu de ramification et $K_{\Delta_{a}}=\Delta_{a} \cap\left(D_{\theta}^{2} \times S_{\eta}^{1}\right)$, 
union $\{0\} \times S_{\eta}^{1}$ (respectivement $\left.S_{\theta}^{1} \times\{0\}\right)$ si $g$ (respectivement $f_{a}$ ) est non réduite, pour valeurs de ramification. Nous construisons la décomposition minimale de Waldhausen de $\left(D_{\theta}^{2} \times S_{\eta}^{1}\right) \backslash\left(\stackrel{\circ}{D_{\alpha}^{2}} \times S_{\eta}^{1}\right)$ qui admet les composantes de $K_{\Delta_{a}} \cup \Phi_{a}\left(K_{f}^{\Sigma_{a}}\right)=K_{\Delta_{a}} \cup\left(\left\{a u^{N}=v\right\} \cap\left(D_{\theta}^{2} \times S_{\eta}^{1}\right)\right)$ pour feuilles. Par prolongement des feuilletages aux tores pleins $D_{\alpha}^{2} \times S_{\eta}^{1}$ et $S_{\theta}^{1} \times D_{\eta}^{2}$, on obtient la décomposition minimale de Waldhausen de $\left(D_{\theta}^{2} \times S_{\eta}^{1}\right) \cup\left(S_{\theta}^{1} \times D_{\eta}^{2}\right)$ qui admet $K_{\Delta_{a}} \cup\left(\left\{a u^{N}=v\right\} \cap\left(D_{\theta}^{2} \times S_{\eta}^{1}\right)\right) \cup\left(\{0\} \times S_{\eta}^{1}\right) \cup\left(S_{\theta}^{1} \times\{0\}\right)$ pour feuilles. Comme les valeurs de ramification de $\Phi_{\mid \Sigma_{a}}$ constituent une réunion de feuilles, le pull-back de la décomposition minimale de Waldhausen de $\left(D_{\theta}^{2} \times S_{\eta}^{1}\right) \cup\left(S_{\theta}^{1} \times D_{\eta}^{2}\right)$, qui a $K_{\Delta_{a}} \cup\left(\left\{a u^{N}=v\right\} \cap\left(D_{\theta}^{2} \times S_{\eta}^{1}\right)\right) \cup\left(\{0\} \times S_{\eta}^{1}\right) \cup$ $\left(S_{\theta}^{1} \times\{0\}\right)$ pour feuilles, fournit une décomposition de Waldhausen (non nécessairement minimale) de $\Sigma_{a}$ pour $f \cdot f_{a} \cdot g \cdot \Gamma_{a}$, à partir de laquelle il est facile d'obtenir une décomposition minimale de Waldhausen de $\Sigma_{a}$ pour $f \cdot f_{a} \cdot g$, comme décrit dans [Ma2] (ou [L-M-W1] pour $g$ forme linéaire transverse à $f$ ).

Remarque 8. - Étant donné une variété de Seifert $\Sigma_{S}$ de la décomposition minimale de Waldhausen de $\Sigma_{a}$ pour $f \cdot f_{a} \cdot g$ décrite ci-dessus, nous posons $V_{S}=\Psi\left(\Sigma_{S}\right)$. Les variétés de Seifert $V_{S}$ ainsi obtenues fournissent une décomposition minimale de Waldhausen de $S_{\varepsilon}^{3}$ pour $f \cdot f_{a} \cdot g$. Notons $G\left(f \cdot f_{a} \cdot g\right)$ le graphe de Waldhausen de cette décomposition.

COROLlaire 3. - 1. $Q_{a} \cup\{N\}=\left\{q_{a}(S)\right.$ où $S$ parcourt les sommets de $\left.G\left(f \cdot f_{a} \cdot g\right)\right\}$

2. $Q_{0} \cup\{N\}=\left\{q(S)\right.$ où $S$ parcourt les sommets de $\left.G\left(f \cdot f_{a} \cdot g\right)\right\}$;

3. Si $S$ est un sommet de $G\left(f \cdot f_{a} \cdot g\right)$ tel que $q(S)<N$ alors $q(S)=q_{a}(S)$.

Démonstration. - Soit $\rho$ une feuille régulière de $V_{S}$. Il existe $i \in\{1, \ldots, k\}$ tel que $\Psi^{-1}(\rho)$ appartienne à $Z_{i}$. Compte-tenu des propriétés du difféomorphisme $\Psi$ et du fait que $\Phi_{a}$ induit un revêtement fini de $\Psi^{-1}(\rho)$ sur $\Phi_{a}\left(\Psi^{-1}(\rho)\right)$, on a :

$\mathcal{L}\left(K_{f_{a}}, \rho\right) / \mathcal{L}\left(K_{g}, \rho\right)=\overline{\mathcal{L}}\left(S_{\theta}^{1} \times\{0\}, \Phi_{a}\left(\Psi^{-1}(\rho)\right)\right) / \overline{\mathcal{L}}\left(\{0\} \times S_{\eta}^{1}, \Phi_{a}\left(\Psi^{-1}(\rho)\right)\right)=$ $p_{i} / q_{i}$, où $\overline{\mathcal{L}}($,$) est le nombre d'enlacement dans la sphère \left(D_{\theta}^{2} \times S_{\eta}^{1}\right) \cup\left(S_{\theta}^{1} \times\right.$ $\left.D_{\eta}^{2}\right)$. D'où le 1 et par symétrie le 2 .

Le 3 est conséquence de 1 et 2 et du fait que si $q(S)<N$ alors $V_{S}$ rencontre au moins une composante $\gamma$ commune à $\Gamma_{0}$ et $\Gamma_{a}$, de sorte que $q(S)=q_{a}(S)$ par la proposition 1 et la proposition a. 


\subsection{Résultats}

Comme au paragraphe 3.2 , nous supposons ici que $N>\min Q_{0}$ et $a \in \mathbb{C} \backslash\{0\}$. Ceci implique que la variété $\Sigma^{N}$, définie ci-dessous, contient $\Phi_{\mid \Sigma_{a}}^{-1}\left(Z_{1}\right)$, et comme dans [Ma1], on peut voir que $\Sigma^{N}$ n'est pas une réunion de tores pleins, voisinage tubulaire de $K_{g}$ dans $\Sigma_{a}$.

DÉfinition 6. - On appelle $\Sigma^{N}$ la réunion des variétés de Seifert $\Sigma_{S}$ de la décomposition minimale de Waldhausen de $\Sigma_{a}$ pour $f \cdot f_{a} \cdot g$ (obtenues en 3.2) qui sont telles que $q(S)<N$ et on pose $W^{N}=\Psi\left(\Sigma^{N}\right)$.

THÉORÈme 3. - Les fibrations $\varphi_{a}=f_{a} /\left|f_{a}\right|_{\mid}: W^{N} \longrightarrow S^{1}$ et $\varphi=$ $f /|f|_{\mid}: W^{N} \longrightarrow S^{1}$ sont isomorphes.

Démonstration. - Le carrousel de Lê (voir [L1]) implique que les fibrations de Milnor pour $f$ et $f_{a}$ se restreignent à $W^{N}$. Donc $\varphi_{a}$ et $\varphi$ sont des fibrations.

Notons $\tau_{j}, 1 \leqslant j \leqslant l$, les composantes de bord des morceaux seifertiques de $W^{N}$.

La fibration $\varphi_{a}$ (resp. $\varphi$ ) induit un homomorphisme $\psi_{a, j}$ (resp. $\psi_{j}$ ) de $H_{1}\left(\tau_{j}, \mathbb{Z}\right)$ sur $\mathbb{Z}$ et un homomorphisme $\psi_{a}$ (resp. $\left.\psi\right)$ de $H_{1}\left(W^{N}, \mathbb{Z}\right)$ sur $\mathbb{Z}$. Si $C$ est une courbe fermée simple sur $\tau_{j}$, alors $\psi_{a, j}(C)$ (resp. $\psi_{j}(C)$ ) est le nombre d'enlacement dans $S_{\varepsilon}^{3}$ de $C$ et de $K_{f_{a}}$ (resp. de $C$ et de $K_{f}$ ) noté $\mathcal{L}\left(C, K_{f_{a}}\right)$ (resp. $\mathcal{L}\left(C, K_{f}\right)$ ).

Comme $W^{N}$ est une variété de Waldhausen dans $S_{\varepsilon}^{3}$, si $\psi_{a, j}=\psi_{j}$ pour tout $j$ dans $\{1, \cdots, l\}$, alors $\psi_{a}=\psi$ et les fibrations $\varphi_{a}$ et $\varphi$ sont isomorphes (voir [E-N], p. 34).

Le théorème 3 se déduit donc du lemme suivant :

LeMme 1. - On a l'égalité $\psi_{a, j}=\psi_{j}$.

Démonstration. - Dans la décomposition de Waldhausen de $S_{\varepsilon}^{3}$ pour $f \cdot f_{a} \cdot g$, construite (en 3.2) à partir du carrousel, $\tau_{j}$ est l'intersection de deux variétés de Seifert $V_{j_{1}}$ et $V_{j_{2}}$ telles que $q_{a}\left(S_{j_{1}}\right)<q_{a}\left(S_{j_{2}}\right) \leqslant N$. D'après le 3 du corollaire 3 et la définition de $W^{N}$, on a $q_{a}\left(S_{j_{1}}\right)=q\left(S_{j_{1}}\right)<q_{a}\left(S_{j_{2}}\right)=$ $q\left(S_{j_{2}}\right) \leqslant N$. Sur $\tau_{j}$ soient $\rho_{1}$ une feuille de Seifert de $V_{j_{1}}$ et $\rho_{2}$ une feuille de Seifert de $V_{j_{2}}$.

On obtient alors $\psi_{a, j}\left(\left[\rho_{1}\right]\right)=q_{a}\left(S_{j_{1}}\right) \cdot \mathcal{L}\left(\rho_{1}, K_{g}\right)=q\left(S_{j_{1}}\right) \cdot \mathcal{L}\left(\rho_{1}, K_{g}\right)=$ $\psi_{j}\left(\left[\rho_{1}\right]\right)$ et de même $\psi_{a, j}\left(\left[\rho_{2}\right]\right)=\psi_{j}\left(\left[\rho_{2}\right]\right)$. 
Comme $\Phi_{a}\left(\Psi^{-1}\left(\rho_{1}\right)\right)$ et $\Phi_{a}\left(\Psi^{-1}\left(\rho_{2}\right)\right)$ sont des feuilles de Seifert qui appartiennent à des zones jacobiennes différentes, leurs classes engendrent un sous-groupe d'indice fini dans $H_{1}\left(\Phi_{a}\left(\Psi^{-1}\left(\tau_{j}\right)\right), \mathbb{Z}\right)$; il en est de même pour les classes de $\rho_{1}$ et $\rho_{2}$ dans $H_{1}\left(\tau_{j}, \mathbb{Z}\right)$, d'où le lemme.

On choisit une fibre $F_{a}$ de la fibration de Milnor $f_{a} /\left|f_{a}\right|: S_{\varepsilon}^{3} \backslash K_{f_{a}} \longrightarrow$ $S^{1}$. On rappelle que $F_{a}^{N}=F_{a} \cap W^{N}$.

Proposition 3. - Le morphisme $H_{1}\left(F_{a}^{N}, \mathbb{Z}\right) \longrightarrow H_{1}\left(F_{a}, \mathbb{Z}\right)$ induit par l'inclusion est injectif.

Démonstration. - Par construction $\Psi^{-1}\left(F_{a}^{N}\right)$ est un morceau de la filtration de la fibre de Milnor obtenue par le carrousel de Lê. Par conséquent (voir [L1]), il existe une monodromie $h: F_{a} \rightarrow F_{a}$ de $f_{a} /\left|f_{a}\right|$ qui se restreint en une monodromie de $\varphi_{a}$ sur $F_{a}^{N}$, et $F_{a}$ est obtenue (à type d'homotopie près) à partir de $F_{a}^{N}$ en ajoutant des anses d'indice un.

Notons $\Lambda_{a}^{N}(t)$ (resp. $\Lambda_{a}(t)$ ) le polynôme caractéristique de l'endomorphisme induit par la monodromie de $\varphi_{a}$ sur $H_{1}\left(F_{a}^{N}, \mathbb{Z}\right.$ ) (resp. par la monodromie de $f_{a} /\left|f_{a}\right|$ sur $\left.H_{1}\left(F_{a}, \mathbb{Z}\right)\right)$. $\Lambda_{0}(t)$.

Corollaire 4. - Pour tout $a \in \mathbb{C}, \Lambda_{a}^{N}(t)=\Lambda_{0}^{N}(t)$ divise $\Lambda_{a}(t)$ et

Dans la section 4.1, on obtient une formule explicite pour $\Lambda_{a}^{N}(t)$ à partir de la résolution minimale de $f_{a} \cdot g$ (voir la proposition 6). Au paragraphe 4.1 nous utiliserons la proposition suivante pour calculer $\Lambda_{a}^{N}(t)$.

Proposition 4. - Soit A une composante connexe de $\overline{\Sigma_{a} \backslash \Sigma^{N}}$ et soit $U_{A}$ un voisinage tubulaire de $A \cap\left(K_{f}^{\Sigma_{a}} \cup K_{f_{a}}^{\Sigma_{a}}\right)$. Alors $\stackrel{\circ}{A}=A \backslash U_{A}$ n'est ni un tore plein ni un tore épaissi.

Démonstration. - Comme $A$ se surjecte par $\Phi_{a} \operatorname{sur}\left(\cup_{j=m+1}^{k} Z_{j}\right) \cup\left(S_{\theta}^{1} \times\right.$ $\left.D_{\eta}^{2}\right), A$ a une composante de bord commune avec $\Sigma^{N}$, et $A$ contient au moins une composante de $K_{f}^{\Sigma_{a}}$ et au moins une composante de $K_{f_{a}}^{\Sigma_{a}}$. Par conséquent, $\stackrel{\circ}{A}$ a au moins trois composantes de bord.

Corollaire 5. - Si une variété de Seifert $V_{S}$ de la décomposition minimale de Waldhausen de $S_{\varepsilon}^{3}$ pour $f \cdot f_{a} \cdot g$ rencontre $K_{f}$ ou $K_{f_{a}}$, alors $q(S) \geqslant N$ et $q_{a}(S) \geqslant N$.

Démonstration. - La décomposition minimale de Waldhausen de $A$ est non triviale et ne contient que des variétés de Seifert $V_{S^{\prime}}$ telles que $q\left(S^{\prime}\right) \geqslant N$ et $q_{a}\left(S^{\prime}\right) \geqslant N$. 
COROLlaIRE 6. - Si $N$ est strictement supérieur au plus grand quotient jacobien de $(g, f)$ alors, pour tout $a \in \mathbb{C}, \Lambda_{a}^{N}(t)=\Lambda_{0}(t)$ divise $\Lambda_{a}(t)$.

Démonstration. - Soit $\stackrel{\circ}{A}$ comme dans la proposition 4 . Notons $\bar{A}$ la variété de Waldhausen obtenue en ajoutant à $\stackrel{\circ}{A}$ les composantes connexes de $U_{A}$ qui contiennent une composante de $K_{f_{a}}^{\Sigma_{a}} ; \bar{A}$ a au moins deux composantes de bord donc ce n'est pas un tore plein.

Si $\bar{A}$ n'était pas un tore épaissi, $\bar{A}$ contiendrait un morceau seifertique essentiel $V$ dont le quotient topologique serait supérieur ou égal à $N$. Par [Ma2] ce quotient serait un quotient jacobien pour $(g, f)$, ce qui contredit l'hypothèse ; $\bar{A}$ est donc un tore épaissi. Donc $\Sigma^{N}$ a le type d'homotopie de $\Sigma_{a} \backslash K_{f}^{\Sigma_{a}}$. La fibration $\varphi$ est alors homotope à la fibration de Milnor de $f$.

\section{Point de vue de la résolution minimale}

\subsection{Résolution et calcul de $\Lambda_{a}^{N}(t)$}

Appelons $\pi$ la résolution minimale de $f \cdot f_{a} \cdot g$ à l'origine de $\mathbb{C}^{2}, a \neq 0$. Le diviseur exceptionnel $\pi^{-1}(0)$ est constitué d'une réunion finie de composantes irréductibles notées $E_{i}, i \in \mathbb{N}$. La transformée totale de $\mathcal{C}=$ $\left(f \cdot f_{a} \cdot g\right)^{-1}(0)$ est $\pi^{-1}(\mathcal{C})$ et la transformée stricte de $\mathcal{C}$ est l'adhérence de $\pi^{-1}(\mathcal{C} \backslash\{0\})$. Une composante irréductible de la transformée stricte intersecte une unique composante $E_{i}$ du diviseur exceptionnel en un unique point.

On définit l'arbre de la résolution minimale de $f \cdot f_{a} \cdot g$, noté $\mathcal{A}\left(f \cdot f_{a} \cdot g\right)$, comme suit.

Chaque $E_{i}$ fournit un sommet $S_{i}$ de l'arbre. Si deux composantes $E_{i}$ et $E_{j}$ s'intersectent alors on relie les sommets $S_{i}$ et $S_{j}$ par une arête. On attache autant de flèches à un sommet $S_{i}$ qu'il existe de composantes irréductibles de la transformée stricte de $\mathcal{C}$ qui rencontrent $E_{i}$.

On factorise en produit de facteurs irréductibles premiers entre eux $f$. $f_{a} \cdot g=\prod_{j=1}^{r} \ell_{j}^{s_{j}}$ et on pondère par $s_{j}$ la flèche qui représente la transformée stricte de $\ell_{j}$.

DÉFINITION 7. - La valuation val $_{E_{i}}(\ell)$ de la composante irréductible $E_{i}$ pour un germe $\ell$ à l'origine de $\mathbb{C}^{2}$ est l'ordre de $\ell \circ \pi$ le long de $E_{i}$.

Pour calculer $\operatorname{val}_{E_{i}}(\ell)$, on considère un germe de courbe lisse $c_{i}$ transverse à $E_{i}$ passant par un point générique $p$ de $E_{i}$. Un tel germe de courbe 
est appelé une curvette de $E_{i}$. On paramétrise $\pi\left(c_{i}\right)$ par $\left(t^{m}, \varphi(t)\right)$. La valuation $\operatorname{val}_{E_{i}}(\ell)$ est la valuation en $t$ de $\ell\left(t^{m}, \varphi(t)\right)$.

Chaque sommet $S_{i}$ de l'arbre $\mathcal{A}\left(f \cdot f_{a} \cdot g\right)$ est pondéré par les deux quotients suivants : $q_{S_{i}}(f)=\operatorname{val}_{E_{i}}(f) / \operatorname{val}_{E_{i}}(g)$ et $q_{S_{i}}\left(f_{a}\right)=\operatorname{val}_{E_{i}}\left(f_{a}\right) / \operatorname{val}_{E_{i}}(g)$. Notons $Q(f)=\left\{q_{S_{i}}(f)\right.$ où $S_{i}$ est un sommet de $\left.\mathcal{A}\left(f \cdot f_{a} \cdot g\right)\right\}$ (resp. $Q\left(f_{a}\right)=$ $\left\{q_{S_{i}}\left(f_{a}\right)\right.$ où $S_{i}$ est un sommet de $\left.\left.\mathcal{A}\left(f \cdot f_{a} \cdot g\right)\right\}\right)$.

Proposition 5. - Si $q_{S_{i}}(f)<N$ alors $q_{S_{i}}\left(f_{a}\right)=q_{S_{i}}(f)<N$, et de même, si $q_{S_{i}}\left(f_{a}\right)<N$ alors $q_{S_{i}}(f)=q_{S_{i}}\left(f_{a}\right)<N$.

Démonstration. - Soit $c_{i}$ est une curvette de $E_{i}$ dont une paramétrisation de Puiseux de $\pi\left(c_{i}\right)$ est donnée par $\left(t^{m}, \varphi(t)\right)$. On a $q_{S_{i}}(f)=\operatorname{val}_{t}$ $\left(f\left(t^{m}, \varphi(t)\right)\right) / \operatorname{val}_{t}\left(g\left(t^{m}, \varphi(t)\right)\right)<N$, d'où :

$$
(*) \operatorname{val}_{t}\left(f\left(t^{m}, \varphi(t)\right)\right)<\operatorname{val}_{t}\left(g\left(t^{m}, \varphi(t)\right)\right) N .
$$

On calcule $q_{S_{i}}\left(f_{a}\right)=\operatorname{val}_{t}\left(f_{a}\left(t^{m}, \varphi(t)\right)\right) / \operatorname{val}_{t}\left(g\left(t^{m}, \varphi(t)\right)\right)$, i.e $q_{S_{i}}\left(f_{a}\right)=$ $\operatorname{val}_{t}\left(f\left(t^{m}, \varphi(t)\right)+a\left(g\left(t^{m}, \varphi(t)\right)\right)^{N}\right) / \operatorname{val}_{t}\left(g\left(t^{m}, \varphi(t)\right)\right)$.

D'après $\left(^{*}\right)$ on obtient $q_{S_{i}}\left(f_{a}\right)=\operatorname{val}_{t}\left(f\left(t^{m}, \varphi(t)\right)\right) / \operatorname{val}_{t}\left(g\left(t^{m}, \varphi(t)\right)\right)$, d'où le résultat.

Même résultat lorsque l'on suppose $\operatorname{val}_{t}\left(f_{a}\left(t^{m}, \varphi(t)\right)\right)<\operatorname{val}_{t}\left(g\left(t^{m}\right.\right.$, $\varphi(t))) N$.

DÉFINITION 8. - La valence $v_{i}$ d'un sommet $S_{i}$ de $\mathcal{A}\left(f \cdot f_{a} \cdot g\right)$ est égale à la somme du nombre d'arêtes et de fièches qui rencontrent $S_{i}$. De plus, $v_{i}(f)$ (resp. $v_{i}\left(f_{a}\right)$ ) est égal à la somme du nombre d'arêtes et de flèches qui représentent des composantes la transformée stricte de $f$ (resp. $f_{a}$ ) et qui rencontrent $S_{i}$.

Remarque 9. - On rappelle que la décomposition minimale de Waldhausen de $S_{\varepsilon}^{3}$ pour $f \cdot f_{a} \cdot g$ peut être obtenue à partir de l'arbre pondéré $\mathcal{A}\left(f \cdot f_{a} \cdot g\right)$. A chaque sommet $S_{i}$ de $\mathcal{A}\left(f \cdot f_{a} \cdot g\right)$ de valence $v_{i} \geqslant 3$ correspond un sommet $S(i)$ de $G\left(f \cdot f_{a} \cdot g\right)$ et on a $q_{a}(S(i))=q_{S_{i}}\left(f_{a}\right)$ et $q(S(i))=q_{S_{i}}(f)$. De plus, si un sommet $S_{i}$ de $\mathcal{A}\left(f \cdot f_{a} \cdot g\right)$ est tel que $v_{i} \geqslant 3$ et $q_{S_{i}}(f)<N$, on a, par le 3 du corollaire $3, q_{S_{i}}\left(f_{a}\right)=q_{S_{i}}(f)<N$ et, par le corollaire 5, $v_{i}\left(f_{a}\right)=v_{i}(f)$. De plus $Q_{0} \subset Q(f)$ et $Q_{a} \subset Q\left(f_{a}\right)$. Donc $\min Q\left(f_{a}\right) \leqslant \min Q_{a}$. $\grave{A}$ partir de maintenant et jusqu'à la fin de 4.4, on étudie les pinceaux avec $N>\min Q(f)$. Vu que $\min Q(f) \leqslant \min Q_{0}$, le cas $N \leqslant \min Q(f)$ fait partie des cas dégénérés traités en 4.5.

Proposition 6. - Supposons $N>\min Q\left(f_{a}\right)$. Appelons $I_{N}$ l'ensemble des indices $i$ des sommets $S_{i}$ de $\mathcal{A}\left(f \cdot f_{a} \cdot g\right)$ tels que $q_{S_{i}}\left(f_{a}\right)<N$ et $D_{N}$ l'ensemble des indices $i$ des sommets $S_{i}$ tels que $q_{S_{i}}\left(f_{a}\right)=N$. 
Le polynôme $\Lambda_{a}^{N}(t)=\left(t^{r_{N}}-1\right) \prod_{i \in I_{N}}\left(t^{v a l_{E_{i}}\left(f_{a}\right)}-1\right)^{v_{i}\left(f_{a}\right)-2}$, où $r_{N}=$ ppcm $_{i \in I_{N} \cup D_{N}}\left(\right.$ val $\left._{E_{i}}\left(f_{a}\right)\right)$, est un facteur commun aux polynômes caractéristiques de la monodromie de $f$ et de $f_{a}$.

Démonstration. - Soit $\tilde{\Sigma}=\left(f_{a} \circ \pi\right)^{-1}\left(S_{\eta}^{1}\right) \cap U$ où $\eta$ est suffisamment petit et $U$ est un "bon" voisinage de $\pi^{-1}(0)$. Soit $\tilde{F}_{a}$ une fibre de Milnor de la fibration $f_{a} \circ \pi_{\mid \tilde{\Sigma}}: \tilde{\Sigma} \longrightarrow S_{\eta}^{1}$. À tout sommet $S_{i} \operatorname{de} \mathcal{A}\left(f \cdot f_{a} \cdot g\right)$ correspond un morceau $F_{i}$ de $\tilde{F}_{a}$ défini comme dans [D-M] section 1.8. On construit une monodromie $h_{a}: \tilde{F}_{a} \longrightarrow \tilde{F}_{a}$ qui se restreint en un difféomorphisme $h_{i}: F_{i} \longrightarrow F_{i}$. On considère maintenant $\tilde{F}_{a}^{N}=\bigcup_{i \in I_{N}} F_{i}$.

La remarque 9 implique que $\tilde{F}_{a}^{N}$ est isotope à la fibre $F_{a}^{N}$ considérée dans la proposition 3 du paragraphe 3.3. Donc le polynôme $\Lambda_{a}^{N}(t)$ est le polynôme caractéristique de l'endomorphisme de $H_{1}\left(\tilde{F}_{a}^{N}, \mathbb{Z}\right)$ induit par la restriction de $h_{a}$ à $\tilde{F}_{a}^{N}$.

On a la suite exacte suivante de Mayer-Vietoris :

$$
\begin{aligned}
& (1) \quad \ldots \longrightarrow 0=H_{2}\left(\tilde{F}_{a}^{N}\right) \longrightarrow \\
& \bigoplus_{\substack{i<j \\
i, j \in I_{N}}} H_{1}\left(F_{i} \cap F_{j}\right) \longrightarrow \oplus_{i \in I_{N}} H_{1}\left(F_{i}\right) \longrightarrow H_{1}\left(\tilde{F}_{a}^{N}\right) \longrightarrow \\
& \bigoplus_{\substack{i<j \\
i, j \in I_{N}}} H_{0}\left(F_{i} \cap F_{j}\right) \longrightarrow \oplus_{i \in I_{N}} H_{0}\left(F_{i}\right) \longrightarrow H_{0}\left(\tilde{F}_{a}^{N}\right) \longrightarrow 0
\end{aligned}
$$

On note respectivement $P_{0}(t), \lambda_{a}^{N}(t), P_{1}(t)$ et $\Lambda_{a}^{N}(t)$ les polynômes caractéristiques de la monodromie sur $\oplus_{i \in I_{N}} H_{0}\left(F_{i}\right), H_{0}\left(\tilde{F}_{a}^{N}\right), \oplus_{i \in I_{N}} H_{1}\left(F_{i}\right)$ et $H_{1}\left(\tilde{F}_{a}^{N}\right)$.

On a donc :

$$
1=\frac{\Lambda_{a}^{N}(t) \cdot P_{0}(t)}{P_{1}(t) \cdot \lambda_{a}^{N}(t)} \text { soit } \Lambda_{a}^{N}(t)=\frac{P_{1}(t) \cdot \lambda_{a}^{N}(t)}{P_{0}(t)} .
$$

$$
\begin{aligned}
& \text { Or } \lambda_{a}^{N}(t)=\left(t^{r_{N}}-1\right), P_{0}(t)=\prod_{i \in I_{N}}\left(t^{r_{i}}-1\right) \text { et } \\
& P_{1}(t)=\prod_{i \in I_{N}}\left(t^{\operatorname{val}_{E_{i}}\left(f_{a}\right)}-1\right)^{v_{i}\left(f_{a}\right)-2}\left(t^{r_{i}}-1\right) .
\end{aligned}
$$

On utilise la proposition 1.7 de [D-M] et le fait que dans [D-M], $D_{i}$ est obtenu à partir de $F_{i}$ en collant un disque à toutes les composantes de bord de $F_{i}$. 
De plus, les valences $\mathrm{v}_{i}$ de $[\mathrm{D}-\mathrm{M}]$ sont les valences $v_{i}\left(f_{a}\right)$ de l'énoncé de la proposition 6 .

LEMME 2 . Si $i \in I_{N}$ alors :

1. $\operatorname{val}_{E_{i}}\left(f_{a}\right)=\operatorname{val}_{E_{i}}(f)$;

2. $v_{i}\left(f_{a}\right)=v_{i}(f)$.

La construction de la monodromie $h_{a}$ décrite ci-dessus est valable pour $a=0$. D'autre part, d'après la proposition $3, \Lambda_{0}^{N}(t)$ divise $\Lambda_{0}(t)$. Par ailleurs, en échangeant le rôle de $a$ et 0 , nous obtenons $\Lambda_{a}^{N}(t)=\Lambda_{0}^{N}(t)$ divise $\Lambda_{0}(t)$ et $\Lambda_{a}(t)$; donc le lemme implique la proposition 6 .

Remarque 10. - D'après le lemme 2, l'arbre $\mathcal{A}\left(f_{a} \cdot g\right)$ permet de déterminer $\Lambda_{a}^{N}(t)$ avec la formule de la proposition 6 .

Démonstration du lemme 2. - La définition de $q_{S_{i}}\left(f_{a}\right)$ et $q_{S_{i}}(f)$ et la proposition 5 impliquent le 1 du lemme.

Si $v_{i} \geqslant 3$, comme $i \in I_{N}$, alors, d'après la remarque $9, v_{i}\left(f_{a}\right)=v_{i}(f)$.

Si $v_{i}=2$, et si $v_{i}\left(f_{a}\right)=1$, alors une composante irréductible de la transformée stricte de $f$ ou de $g$ rencontre $E_{i}$. Si c'est une composante de $f$, on a $q_{S_{i}}\left(f_{a}\right)=N$, ce qui est exclu. Si c'est une composante de $g$, on a $v_{i}\left(f_{a}\right)=v_{i}(f)=1$.

De même, $v_{i}(f)=1$ implique $v_{i}\left(f_{a}\right)=1$.

Sinon, $v_{i}\left(f_{a}\right)=v_{i}(f)=2$.

Si $v_{i}=1$ alors $v_{i}\left(f_{a}\right)=v_{i}(f)=1$.

\subsection{Sommets dicritiques}

Le cas $g(x, y)=x$ est particulièrement important puisqu'il s'applique à l'étude des valeurs atypiques à l'infini des applications polynômiales de $\mathbb{C}^{2}$ dans $\mathbb{C}$. C'est pourquoi nous établissons des formules précises pour de telles familles de pinceaux. Elles se généralisent facilement lorsque $g$ est irréductible. On suppose de plus $N>\min Q(f)$. Le cas $N \leqslant \min Q(f)$ sera traité en 4.5 .

On convient de noter $S_{0}$ le sommet de $\mathcal{A}\left(f \cdot f_{a} \cdot x\right)$ où s'accroche la flèche qui représente la transformée stricte de $x$. On oriente les arêtes de $\mathcal{A}\left(f \cdot f_{a} \cdot x\right)$ à partir du sommet $S_{0}$. On colorie en rouge (resp. bleu) les géodésiques orientées qui vont de $S_{0}$ à l'extrémité des flèches qui représentent les composantes de la transformée stricte de $f_{a}$ (resp. $f$ ). L'arbre $\mathcal{A}\left(f \cdot f_{a} \cdot x\right)$ ainsi obtenu est pondéré, orienté et coloré. 
Fibrations associées à un pinceau de courbes planes

Un sommet $S_{i}$ de $\mathcal{A}\left(f \cdot f_{a} \cdot x\right)$ est de rupture pour $f_{a}$ (resp. $\left.f\right)$ si $v_{i}\left(f_{a}\right)$ (resp. $\left.v_{i}(f)\right)$ est supérieur ou égal à trois lorsque $i \neq 0$, et $S_{0}$ est un sommet de rupture pour $f_{a}$ (resp. $f$ ) si $v_{0}\left(f_{a}\right)$ (resp. $v_{0}(f)$ ) est supérieur ou égal à deux.

Notons $R\left(f_{a}\right)$ (resp. $R(f)$ ) l'ensemble des indices $i$ de $S_{i}$ qui sont tels que $S_{i}$ est un sommet de rupture pour $f_{a}$ (resp.f).

Pour une démonstration des deux résultats suivants on pourra consulter [L-M-W2] dans le cas où $x$ est transverse à $f$ ou [Ma1] pour le cas général.

Proposition d. - L'ensemble des quotients $q_{S_{i}}\left(f_{a}\right), i \in R\left(f_{a}\right)$ (resp. $q_{S_{i}}(f), i \in R(f)$ ) est l'ensemble des quotients polaires de $f_{a}$ (resp. $f$ ) pour la direction $x$.

THÉORÈME DE CROISSANCE. - Il y a croissance stricte de $q_{S_{i}}\left(f_{a}\right)$ (resp. $\left.q_{S_{i}}(f)\right)$ le long des géodésiques de $f_{a}$ (resp. $f$ ) et constance sur les arêtes incolores ou unicolores bleues (resp. incolores ou unicolores rouges). En particulier $q_{S_{0}}(f)=\min Q(f)\left(\right.$ resp. $\left.q_{S_{0}}\left(f_{a}\right)=\min Q\left(f_{a}\right)\right)$.

Comme on a supposé $N>\min Q(f)$, on a donc $q_{S_{0}}(f)<N$, et $q_{S_{0}}\left(f_{a}\right)=$ $q_{S_{0}}(f)<N$.

Proposition 7. - Soit $S_{i}$ un sommet bicolore de $A\left(f \cdot f_{a} \cdot x\right)$ dont au moins une arête (ou flèche) unicolore est issue. Alors aucune arête sortante de $S_{i}$ n'est bicolore et $q_{S_{i}}\left(f_{a}\right)=q_{S_{i}}(f)=N$.

Démonstration. - On suppose que $S_{i}$ est un sommet bicolore auquel est attachée une arête (ou une flèche) unicolore bleue. Choisissons une curvette $c$ qui passe par le point singulier de la transformée totale de $\mathcal{C}$ symbolisé par l'arête ou la flèche bleue. Cette curvette est générique pour $f_{a} \cdot x$. Soit $\left(t^{m}, \varphi(t)\right)$ une paramétrisation de $\pi(c)$.

Soit $c_{1}$ une curvette de $S_{i}$ générique pour $f \cdot x$ et pour $f_{a}$. Soit $\left(t^{m_{1}}, \varphi_{1}(t)\right)$ une paramétrisation de $\pi\left(c_{1}\right)$. Notons que $m=m_{1}$ car $c$ et $c_{1}$ sont les curvettes d'un même sommet, génériques pour $x$.

Par choix de $c$ et $c_{1}$ on a :

$$
\begin{gathered}
\operatorname{val}_{t}\left(f\left(t^{m}, \varphi(t)\right)\right)>\operatorname{val}_{t}\left(f\left(t^{m}, \varphi_{1}(t)\right)\right) \text { et } \\
\operatorname{val}_{t}\left(f_{a}\left(t^{m}, \varphi(t)\right)\right)=\operatorname{val}_{t}\left(f_{a}\left(t^{m}, \varphi_{1}(t)\right)\right) .
\end{gathered}
$$

$\operatorname{Donc}_{\operatorname{val}_{t}}\left(f\left(t^{m}, \varphi(t)\right)+a t^{m N}\right)=\operatorname{val}_{t}\left(f\left(t^{m}, \varphi_{1}(t)\right)+a t^{m N}\right)$ et par conséquent on obtient :

$$
\begin{gathered}
\operatorname{val}_{t}\left(f_{a}\left(t^{m}, \varphi_{1}(t)\right)\right)=m N \text { et } \\
-763-
\end{gathered}
$$




$$
\operatorname{val}_{t}\left(f\left(t^{m}, \varphi_{1}(t)\right)\right) \geqslant m N
$$

Ainsi on a $q_{S_{i}}\left(f_{a}\right)=N$ et $q_{S_{i}}(f) \geqslant N$.

Choisissons désormais une curvette $c_{2}$ passant par le point singulier de la transformée totale de $\mathcal{C}$ symbolisé par une arête (ou flèche) rouge (unicolore ou bicolore). Soit $\left(t^{m}, \varphi_{2}(t)\right)$ une paramétrisation de $\pi\left(c_{2}\right)$. On a forcément $\operatorname{val}_{t}\left(f_{a}\left(t^{m}, \varphi_{2}(t)\right)\right)>m N$ i.e. $\operatorname{val}_{t}\left(f\left(t^{m}, \varphi_{2}(t)\right)+a t^{m N}\right)>m N$, ce qui implique $f\left(t^{m}, \varphi_{2}(t)\right)=-a t^{m N}+\cdots$ et par conséquent $\operatorname{val}_{t}\left(f\left(t^{m}, \varphi_{2}(t)\right)=m N\right.$ donc $q_{S_{i}}(f)=N$. Ceci prouve également que $c_{2}$ est générique pour $f$ et par conséquent que l'arête rouge est unicolore, ce qui achève la démonstration.

Proposition 8. - Si $S_{j}$ est un sommet de $\mathcal{A}\left(f \cdot f_{a} \cdot x\right)$ tel que $q_{S_{j}}\left(f_{a}\right)=$ $q_{S_{j}}(f)=N$, alors aucune arête sortante de $S_{j}$ n'est bicolore.

Démonstration. - Supposons qu'une arête bicolore sorte par $S_{j}$. On choisit une géodésique, par exemple bleue, qui passe par cette arête. Elle aboutit à une flèche bleue. Soit $S_{i}$ le dernier sommet bicolore sur cette géodésique. La géodésique choisie sort de $S_{i}$ par une arête (ou flèche) unicolore bleue. Par la proposition 7 , on a donc $q_{S_{i}}\left(f_{a}\right)=q_{S_{i}}(f)=N$. Comme $S_{i}$ se trouve après $S_{j}$, ceci contredit le théorème de croissance.

Voici désormais quelques conséquences de ces résultats.

Corollaire 7. - Dans $\mathcal{A}\left(f \cdot f_{a} \cdot x\right)$, sur toute géodésique de $f$, il existe un unique sommet $S_{i_{0}}$ de quotient $N$ qui est un sommet bicolore dont toutes les arêtes sortantes sont unicolores, et tel que $q_{S_{i_{0}}}\left(f_{a}\right)=q_{S_{i_{0}}}(f)=N$. Un tel sommet $S_{i_{0}}$ est dit "dicritique".

Sur cette géodésique, les sommets précédant $S_{i_{0}}$ sont bicolores et de quotient strictement inférieur à $N$ pour $f$ et $f_{a}$. Les sommets succédant $\grave{a} S_{i_{0}}$ sont unicolores de quotient égal à $N$ pour $f_{a}$, et de quotient strictement supérieur à $N$ pour $f$.

CoRollaire 8. - Si $S_{i}$ est un sommet de $\mathcal{A}\left(f \cdot f_{a} \cdot x\right)$ avec $q_{S_{i}}(f)<N$, alors (on a $q_{S_{i}}(f)=q_{S_{i}}\left(f_{a}\right)$ ) aucune arête ou flèche unicolore ne rencontre $S_{i}$.

DÉFINITION 9. - On définit $\mathcal{A}_{N}\left(f \cdot f_{a} \cdot x\right)$ (respectivement $\mathcal{A}_{N}(f \cdot x)$ ) le sous-graphe de $\mathcal{A}\left(f \cdot f_{a} \cdot x\right)$ (respectivement $\mathcal{A}(f \cdot x)$ ) constitué des sommets $S_{i}$ de $\mathcal{A}\left(f \cdot f_{a} \cdot x\right)$ (respectivement $\mathcal{A}(f \cdot x)$ ) qui sont tels que $q_{S_{i}}(f)<N$, de toutes les arêtes attachées à ces sommets et de la flèche qui représente la transformée stricte de $x$. 
REMARQUE 11. - Une flèche privée de sa pointe est considérée comme une arête.

Corollaire 9. - Dans $\mathcal{A}\left(f \cdot f_{a} \cdot x\right)$ on $a:$

(i) $\mathcal{A}_{N}\left(f \cdot f_{a} \cdot x\right)$ est connexe ; c'est donc un sous-arbre.

(ii) $\mathcal{A}_{N}(f \cdot x)$ est un sous-arbre de $\mathcal{A}_{N}\left(f \cdot f_{a} \cdot x\right)$.

Démonstration. - La croissance stricte des quotients polaires sur les géodésiques colorées et leur constance sur les arêtes incolores prouve que $\mathcal{A}_{N}\left(f \cdot f_{a} \cdot x\right)$ est connexe.

Comme $\mathcal{A}_{N}\left(f \cdot f_{a} \cdot x\right)$ ne contient aucune arête ou flèche unicolore et que $\mathcal{A}\left(f \cdot f_{a} \cdot x\right)$ est l'arbre de la résolution minimale de $f \cdot f_{a} \cdot x$, on a (ii).

\subsection{Multiplicité sortante pour un dicritique}

Préliminaires. - Soit $\ell$ un germe de fonction analytique, $\mathcal{A}(\ell)$ un arbre d'une résolution de $\ell$ à l'origine de $\mathbb{C}^{2}$, et soit $x$ un axe qui n'est pas une composante de $\ell$. Comme précédemment, on note $S_{0}$ le sommet où s'accroche la transformée stricte de $x$. On oriente $\mathcal{A}(\ell)$ à partir de $S_{0}$. Soit $S_{i}$ un sommet de $\mathcal{A}(\ell)$ et soit $\varepsilon$ une arête issue de $S_{i}$. On note $S_{i+1}$ l'autre extrémité de $\varepsilon$.

Soit $c_{i}$ (respectivement $c_{i+1}$ ) une curvette de la composante irréductible du diviseur exceptionnel correspondant à $S_{i}$ (respectivement $S_{i+1}$ ); on dira que $c_{i}$ est associée à $S_{i}$. Soit $z$ un axe transverse à $x \cdot \ell$. Un développement de Puiseux de $\pi\left(c_{i}\right)$ est donné par :

$$
z=\sum_{j=1}^{k} a_{j} x^{m_{j} / n_{1} \ldots n_{j}}+b_{i} x^{m_{i} / n_{1} \ldots n_{k} n_{i}}
$$

Un développement de Puiseux de $\pi\left(c_{i+1}\right)$ est :

cas I :

$$
z=\sum_{j=1}^{k} a_{j} x^{m_{j} / n_{1} \ldots n_{j}}+b x^{m_{i} / n_{1} \ldots n_{k} n_{i}}+b_{i+1} x^{m_{i+1} / n_{1} \ldots n_{k} n_{i} n_{i+1}},
$$

cas II :

$$
z=\sum_{j=1}^{k} a_{j} x^{m_{j} / n_{1} \ldots n_{j}}+b_{i+1} x^{m_{i+1} / n_{1} \ldots n_{k} n_{i+1}}, \text { avec }
$$


Hélène Maugendre, Françoise Michel

$$
\begin{aligned}
\frac{m_{j}}{n_{1} \ldots n_{j}} & <\frac{m_{j+1}}{n_{1} \ldots n_{j+1}}<\ldots<\frac{m_{i}}{n_{1} \ldots n_{k} n_{i}}<\frac{m_{i+1}}{n_{1} \ldots n_{k} n_{i} n_{i+1}} \text { pour le cas I et } \\
\frac{m_{j}}{n_{1} \ldots n_{j}} & <\frac{m_{j+1}}{n_{1} \ldots n_{j+1}}<\ldots<\frac{m_{i}}{n_{1} \ldots n_{k} n_{i}}<\frac{m_{i+1}}{n_{1} \ldots n_{k} n_{i+1}} \text { pour le cas II }
\end{aligned}
$$

les $n_{j}, n_{i}, n_{i+1}$ pouvant être égaux à un et $a_{j}, b_{i}, b, b_{i+1}$ étant des nombres complexes non nuls.

DÉFINITION 10. - Le sommet $S_{i}$ est caractéristique pour $\pi\left(c_{i+1}\right)$ si et seulement si $n_{i}>1$ et $b \neq 0$, i.e. dans le cas $I$ et $n_{i}>1$. Sinon $S_{i}$ n'est pas caractéristique pour $\pi\left(c_{i+1}\right)$.

DÉFINITION 11. - Si $c_{i}^{\prime}$ est une autre curvette associée à $S_{i}$, on pose :

$$
l_{i}=\frac{\left(\pi\left(c_{i}\right), \pi\left(c_{i}^{\prime}\right)\right)_{0}}{n_{i}} .
$$

Le couple d'entiers $\left(l_{i}, n_{i}\right)$ (p.g.c.d $\left(l_{i}, n_{i}\right)=1$ ) est la paire associée au sommet $S_{i}$.

REMARQUE 12. - Nous rappelons que les $l_{i}$ satisfont les formules suivantes (pour plus de détails voir [M-W] chapitre 5):

(i) Si aucun sommet caractéristique pour $\pi\left(c_{i}\right)$ ne précède $S_{i}$ sur la géodésique de $\pi\left(c_{i}\right)$, alors $l_{i}=m_{i}$.

(ii) Sinon, $l_{i}=m_{i}+n_{i}(l n-m)$, où $(l, n)$ est le couple associé au dernier sommet caractéristique pour $\pi\left(c_{i}\right)$ qui précède $S_{i}$ sur la géodésique de $\pi\left(c_{i}\right)$.

Rappelons la proposition 5.4.1 du chapitre 5 de [M-W] qui nous sera utile pour ce qui suit.

Proposition e. - Soient $\ell_{1}$ et $\ell_{2}$ deux composantes irréductibles d'un germe de fonction analytique $\ell$ à l'origine de $\mathbb{C}^{2}$. Désignons par $\left(l_{i}, n_{i}\right)$, le couple d'entiers associé au sommet $S_{i}$ où se séparent les géodésiques de $\ell_{1}$ et $\ell_{2}$. Avec les notations précédentes nous avons :

$$
\left(\ell_{1}, \ell_{2}\right)_{0}=\left(\ell_{1}, x\right)_{0} \cdot\left(\ell_{2}, x\right)_{0} \cdot \frac{l_{i}}{\left(n_{1} \cdots n_{k}\right)^{2} n_{i}} .
$$

Notation. - On factorise $\ell$ en $\ell=\ell_{\varepsilon} \cdot \ell_{\tilde{\varepsilon}}$ où les géodésiques des facteurs de $\ell_{\varepsilon}$ (respectivement $\ell_{\tilde{\varepsilon}}$ ) passent par l'arête $\varepsilon$ (respectivement ne passent pas par $\varepsilon)$.

DÉFINITION 12. - La multiplicité sortante de $\ell$ par l'arête $\varepsilon$, notée $m_{\varepsilon}(\ell)$ est égale $\grave{a}$ :

$$
\frac{\left(\ell_{\varepsilon}, \pi\left(c_{i}\right)\right)_{0}}{l_{i} n_{i}} \text { pour le cas I et } \frac{\left(\ell_{\varepsilon}, \pi\left(c_{i}\right)\right)_{0}}{l_{i}} \text { pour le cas II. }
$$


Proposition 9. - Soit $S_{i}$ un sommet de $\mathcal{A}\left(f \cdot f_{a} \cdot x\right)$. Si $q_{S_{i}}(f)<N$ et si $\varepsilon$ est une arête de $\mathcal{A}\left(f \cdot f_{a} \cdot x\right)$ qui a pour origine $S_{i}$ et pour extrémité $S_{i+1}$, alors $m_{\varepsilon}(f)=m_{\varepsilon}\left(f_{a}\right)$ et $\left(x, f_{\varepsilon}\right)_{0}=\left(x, f_{a, \varepsilon}\right)_{0}$.

Démonstration. - Comme pour tout $b \in \mathbb{C}$ on a $\left(x, f_{b, \varepsilon}\right)_{0}=m_{\varepsilon}\left(f_{b}\right) n_{1} \ldots n_{k}$, il suffit de vérifier que pour tout $a \in \mathbb{C}$ on a $m_{\varepsilon}(f)=m_{\varepsilon}\left(f_{a}\right)$.

D'après la proposition 5 , on a $q_{S_{i}}(f)=q_{S_{i}}\left(f_{a}\right)$. Par conséquent, si $c_{i}$ désigne une curvette de $S_{i}$, on obtient pour le cas I :

$$
\begin{aligned}
\frac{\left(\pi\left(c_{i}\right), f_{\varepsilon}\right)_{0}+\left(\pi\left(c_{i}\right), f_{\tilde{\varepsilon}}\right)_{0}}{\left(\pi\left(c_{i}\right), x\right)_{0}} & =\frac{\left(\pi\left(c_{i}\right), f_{a, \varepsilon}\right)_{0}+\left(\pi\left(c_{i}\right), f_{a, \tilde{\varepsilon}}\right)_{0}}{\left(\pi\left(c_{i}\right), x\right)_{0}}, \text { soit } \\
\frac{m_{\varepsilon}(f) \cdot l_{i} \cdot n_{i}+\left(\pi\left(c_{i}\right), f_{\tilde{\varepsilon}}\right)_{0}}{\left(\pi\left(c_{i}\right), x\right)_{0}} & =\frac{m_{\varepsilon}\left(f_{a}\right) \cdot l_{i} \cdot n_{i}+\left(\pi\left(c_{i}\right), f_{a, \tilde{\varepsilon}}\right)_{0}}{\left(\pi\left(c_{i}\right), x\right)_{0}}, \text { d'où } \\
m_{\varepsilon}(f) \cdot l_{i} \cdot n_{i}+\left(\pi\left(c_{i}\right), f_{\tilde{\varepsilon}}\right)_{0} & =m_{\varepsilon}\left(f_{a}\right) \cdot l_{i} \cdot n_{i}+\left(\pi\left(c_{i}\right), f_{a, \tilde{\varepsilon}}\right)_{0} \quad \text { (1). }
\end{aligned}
$$

Par ailleurs comme $q_{S_{i}}(f)<N$, d'après le corollaire 7 , on sait que le sommet $S_{i+1}$ est tel que $q_{S_{i+1}}(f)=q_{S_{i+1}}\left(f_{a}\right) \leqslant N$. Pour ce sommet, $q_{S_{i+1}}(f)=q_{S_{i+1}}\left(f_{a}\right)$, donc d'après la proposition e, ceci équivaut à $m_{\varepsilon}(f)$. $l_{i+1}+n_{i+1}\left(\pi\left(c_{i}\right), f_{\tilde{\varepsilon}}\right)_{0}=m_{\varepsilon}\left(f_{a}\right) \cdot l_{i+1}+n_{i+1}\left(\pi\left(c_{i}\right), f_{a, \tilde{\varepsilon}}\right)_{0} \quad$ (2).

Avec (1) on a : $\left(\pi\left(c_{i}\right), f_{\tilde{\varepsilon}}\right)_{0}-\left(\pi\left(c_{i}\right), f_{a, \tilde{\varepsilon}}\right)_{0}=\left(m_{\varepsilon}\left(f_{a}\right)-m_{\varepsilon}(f)\right) l_{i} \cdot n_{i}$, et avec (2), $n_{i+1} \cdot l_{i} \cdot n_{i}\left(m_{\varepsilon}\left(f_{a}\right)-m_{\varepsilon}(f)\right)=l_{i+1}\left(m_{\varepsilon}\left(f_{a}\right)-m_{\varepsilon}(f)\right)$. Ainsi on obtient $n_{i+1} \cdot l_{i} \cdot n_{i}=l_{i+1} \quad(*)$.

Ceci implique que $n_{i+1}$ divise $l_{i+1}$ et donc $n_{i+1}$ divise $m_{i+1}$. Par conséquent $n_{i+1}=1$. Considérons désormais deux cas : celui où $n_{i}=1$ puis celui où $n_{i}>1$.

Dans le premier cas $(*)$ devient $m_{i+1}+(l n-m)=m_{i}+(l n-m)$, où $(l, n)$ est le couple associé au sommet caractéristique pour $\pi\left(c_{i}\right)$ qui précède $S_{i}$ (s'il n'existe pas de tel sommet de rupture, dans les formules cela revient à prendre $l=m$ et $n=1$ ). On obtient alors $m_{i}=m_{i+1}$, ce qui est impossible.

Dans le second cas, $(*)$ devient $: m_{i+1}+\left(l_{i} n_{i}-m_{i}\right)=l_{i} n_{i}$. On obtient encore $m_{i}=m_{i+1}$, ce qui est absurde.

Pour le cas II nous avons $m_{\varepsilon}(f) \cdot l_{i}+\left(\pi\left(c_{i}\right), f_{\tilde{\varepsilon}}\right)_{0}=m_{\varepsilon}\left(f_{a}\right) \cdot l_{i}+\left(\pi\left(c_{i}\right), f_{a, \tilde{\varepsilon}}\right)_{0}$ $\left(1^{\prime}\right)$ et

$m_{\varepsilon}(f) \cdot l_{i+1}+\frac{n_{i+1}}{n_{i}}\left(\pi\left(c_{i}\right), f_{\tilde{\varepsilon}}\right)_{0}=m_{\varepsilon}\left(f_{a}\right) \cdot l_{i+1}+\frac{n_{i+1}}{n_{i}}\left(\pi\left(c_{i}\right), f_{a, \tilde{\varepsilon}}\right)_{0} \quad\left(2^{\prime}\right)$.

Avec $\left(1^{\prime}\right)$ et $\left(2^{\prime}\right)$ on obtient alors l'égalité suivante $: l_{i+1} \cdot n_{i}=l_{i}$. $n_{i+1}(* *)$. 
Nous allons considérer deux cas, suivant qu'il existe un sommet caractéristique pour $\pi\left(c_{i}\right)$ qui précède $S_{i}$ ou pas.

S'il n'existe pas de tel sommet, on a $l_{i+1}=m_{i+1}$ et $l_{i}=m_{i}$. L'égalité (**) devient alors $m_{i} n_{i+1}=m_{i+1} n_{i}$, ce qui équivaut à $m_{i} / n_{i}=m_{i+1} / n_{i+1}$, ce qui est impossible.

S'il existe un sommet de rupture qui précède $S_{i}$, avec les notations qui précèdent, $(* *)$ s'écrit : $\left(m_{i}+n_{i}(l n-m)\right) n_{i+1}=\left(m_{i+1}+n_{i+1}(l n-m)\right) n_{i}$, soit $m_{i} / n_{i}=m_{i+1} / n_{i+1}$, ce qui est impossible.

Avec les mêmes notations que dans la proposition 9 nous avons :

CoRollaire 10. - (i) $\left(f_{a, \tilde{\varepsilon}}, \pi\left(c_{i}\right)\right)_{0}=\left(f_{\tilde{\varepsilon}}, \pi\left(c_{i}\right)\right)_{0}$;

(ii) $\left(x, f_{\tilde{\varepsilon}}\right)_{0}=\left(x, f_{a, \tilde{\varepsilon}}\right)_{0}$;

Démonstration. - $(i)$ est une conséquence directe de la proposition 9 et de la définition de $m_{\varepsilon}(f)$ et $m_{\varepsilon}\left(f_{a}\right)$. Pour $(i i)$ on utilise le fait que $q_{S_{0}}(f)=$ $q_{S_{0}}\left(f_{a}\right)<N$, ce qui implique que $(x, f)_{0}=\left(x, f_{a}\right)_{0}$. On conclut en utilisant la proposition 9 .

\subsection{Détermination de $\mathcal{A}\left(f \cdot f_{a} \cdot x\right)$ en fonction de $\mathcal{A}(f \cdot x)$}

THÉORÈME 4. - Lorsque a est régulière, $\mathcal{A}\left(f_{a} \cdot x\right)$ s'obtient à partir de $\mathcal{A}(f \cdot x)$ comme suit.

(i) On ajoute à $\mathcal{A}_{N}(f \cdot x)$ tous les sommets de quotient $N$ de $\mathcal{A}(f \cdot x)$. On note $\mathcal{A}_{N}^{\prime}(f \cdot x)$ l'arbre ainsi obtenu.

(ii) Pour chaque arête semi-ouverte $\varepsilon$ sortante de $\mathcal{A}_{N}(f \cdot x)$, on note $S_{\varepsilon}$ son sommet origine.

a Si l'extrémité de $\varepsilon$ dans $\mathcal{A}(f \cdot x)$ est une pointe de flèche de poids un, alors on ajoute cette pointe.

$b$ Si l'extrémité de $\varepsilon$ dans $\mathcal{A}(f \cdot x)$ est un sommet $S$ de quotient $N$ pour $f$ et de paire $\left(l_{S}, n_{S}\right)$, on accroche $m_{\varepsilon}(f) / n_{S}$ flèches de poids un à $S$ dans $\mathcal{A}_{N}^{\prime}(f \cdot x)$.

c Si l'extrémité de $\varepsilon$ dans $\mathcal{A}(f \cdot x)$ est une pointe de flèche de poids strictement supérieur à un ou un sommet $S$ de quotient strictement supérieur à $N$ pour $f$, alors on raccroche à $\varepsilon$ une zone possédant exactement un sommet de rupture $S^{\prime}$ de quotient $N$ pour $f_{a}$, et on 
ajoute $m_{\varepsilon}(f) / n_{S^{\prime}}$ flèches à $S^{\prime}$. La paire $\left(l_{S^{\prime}}, n_{S^{\prime}}\right)$ associée à $S^{\prime}$ est obtenue comme suit. Posons :

$$
\omega=\frac{N v a l_{E_{\varepsilon}}(x)-v a l_{E_{\varepsilon}}\left(f_{\tilde{\varepsilon}}\right)}{m_{\varepsilon}(f)}
$$

( $\alpha)$ Si $S_{\varepsilon}$ est un sommet caractéristique pour les branches de $f_{\varepsilon}$, on a $l_{S^{\prime}} / n_{S^{\prime}}=\omega$.

( $\beta$ ) Sinon, $l_{S^{\prime}} / n_{S^{\prime}}=\omega / n_{S \varepsilon}$.

(iii) On efface la couleur bleu et on colorie en rouge les géodésiques des composantes irréductibles de $f_{a}$.

THÉORÈME 5. - Lorsque a est régulière, on construit l'arbre $\mathcal{A}\left(f \cdot f_{a} \cdot x\right)$ comme suit. On prend l'arbre $\mathcal{A}\left(f_{a} \cdot x\right)$ et les composantes connexes de $\mathcal{A}(f \cdot x) \backslash \mathcal{A}_{N}^{\prime}(f \cdot x)=\coprod_{i=1}^{p} A_{i}$.

a Si $A_{i}$ est une pointe de flèche de poids un, dans $\mathcal{A}\left(f_{a} \cdot x\right)$ on remplace l'arête $\varepsilon$ et la pointe de fièche accrochée à $\varepsilon$ par une zone de la forme

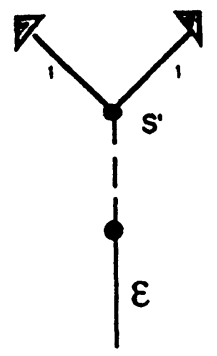

où le sommet $S^{\prime}$ est dicritique, de paire associée $\left(l_{S^{\prime}}, n_{S^{\prime}}\right)$ avec $n_{S^{\prime}}=1$ et $l_{S^{\prime}}=\omega$, et le nombre de sommets ajoutés sur la géodésique issue de $S_{\varepsilon}$ est égal à $l_{S^{\prime}}-l_{S_{\varepsilon}} n_{S_{\varepsilon}}$.

b Si $A_{i}$ a une arête origine $\varepsilon_{i}$ semi-ouverte, alors son sommet origine $S$ dans $\mathcal{A}(f \cdot x)$ est tel que $q_{S}(f)=N$, et alors dans $\mathcal{A}\left(f_{a} \cdot x\right)$ on raccroche $A_{i}$ au sommet $S$ de $\mathcal{A}\left(f_{a} \cdot x\right)$.

c (1) Si $A_{i}$ est une pointe de flèche de poids $r_{i}$ strictement supérieur à un, on ajoute dans $\mathcal{A}\left(f_{a} \cdot x\right)$ une flèche de poids $r_{i}$ à $S^{\prime}$ si $n_{S^{\prime}}=1$ (où $S^{\prime}$ est le sommet de quotient $N$ défini au (c) du théorème 4) ou au sommet de valence un, extrémité de la zone de rupture associée à $S^{\prime}$ si $n_{S^{\prime}}>1$.

(2) Sinon, $A_{i}$ a un sommet origine $S^{\prime \prime}$ et $q_{S^{\prime \prime}}(f)>N$. Si $n_{S^{\prime}}=1$, on raccroche $A_{i} \grave{a} \mathcal{A}\left(f_{a} \cdot x\right)$ en mettant une arête entre $S^{\prime}$ et $S^{\prime \prime}$ (où $S^{\prime}$ 
est le sommet de quotient $N$ défini au (c) du théorème 4). Si $n_{S^{\prime}}>1$, on insère dans $A_{i}$ la partie incolore de $\mathcal{A}\left(f_{a} \cdot x\right)$ qui s'accroche à $S^{\prime}$ en tenant compte de la résolution des composantes de $f_{\varepsilon}$.

On colorie en rouge et bleu en suivant les géodésiques des composantes irréductibles de $f_{a}$ et de $f$ respectivement.

Remarque 13. - (i) Dans le cas a des théorèmes 4 et 5 , on a $m_{\varepsilon}(f)=$ 1. Si $S_{\varepsilon}$ n'est pas caractéristique pour la composante de $f_{\varepsilon}$, on a $n_{S_{\varepsilon}}=1$ car $\varepsilon$ est un support pour la flèche qui représente $f_{\varepsilon}$.

(ii) Le cas $n_{S^{\prime}}>1$ du c(2) du théorème 5 se fait explicitement en utilisant les paires de Zariski des branches de $f_{\varepsilon}^{-1}(0)$ comme dans [M-W] chapitre 6. Avec les arbres topologiques de satellisation, on raccrocherait directement le bout d'arbre correspondant à $A_{i}$ à la fourche correspondant à $S^{\prime}$ (voir [M-W] chapitre 3).

Démonstration des théorèmes 4 et 5 . - Nous avons l'inclusion $\mathcal{A}_{N}(f$. $x) \subset \mathcal{A}\left(f \cdot f_{a} \cdot x\right)$.

Soit $\varepsilon$ une arête sortante de $\mathcal{A}_{N}(f \cdot x)$.

Si l'extrémité $S$ de $\varepsilon$ dans $\mathcal{A}(f \cdot x)$ est un sommet de quotient $N$, alors $f_{a, \varepsilon}$ correspond à des curvettes de $S$, et les b des théorèmes 4 et 5 sont évidents.

Si l'extrémité $S$ de $\varepsilon$ dans $\mathcal{A}(f \cdot x)$ est une pointe de flèche de poids un, alors on a $m_{\varepsilon}(f)=1=m_{\varepsilon}\left(f_{a}\right)$, et donc $f_{a, \varepsilon}$ (et $f_{\varepsilon}$ ) est irréductible et sa géodésique dans $\mathcal{A}\left(f \cdot f_{a} \cdot x\right)$ ne contient pas de sommet caractéristique après $S_{\varepsilon}$ (d'après la remarque $13(\mathrm{i})$ ).

Par conséquent le a du théorème 4 est clair.

Pour le a du théorème 5 , on a ajouté le sommet $S^{\prime}$ de quotient $N$, de paire $\left(l_{S^{\prime}}, n_{S^{\prime}}\right)$ avec $n_{S^{\prime}}=1$ (par la remarque 13 (i)), et $f_{a, \varepsilon}$ et $f_{\varepsilon}$ sont des curvettes de $S^{\prime}$. D'après la définition 11 , on a donc $l_{S^{\prime}}=\left(f_{a, \varepsilon}, f_{\varepsilon}\right)_{0}$, et on obtient :

$$
N=\frac{\left(f_{a}, f_{\varepsilon}\right)_{0}}{\left(x, f_{\varepsilon}\right)_{0}}=\frac{\left(f, f_{a, \varepsilon}\right)_{0}}{\left(x, f_{\varepsilon}\right)_{0}}=\frac{\left(f_{\varepsilon}, f_{a, \varepsilon}\right)_{0}+\left(f_{\tilde{\varepsilon}}, f_{a, \varepsilon}\right)_{0}}{\left(x, f_{\varepsilon}\right)_{0}}
$$

$\operatorname{car}\left(x, f_{\varepsilon}\right)_{0}=\left(x, f_{a, \varepsilon}\right)_{0}=\operatorname{val}_{E^{\prime}}(x)$, d'où l'on tire $N\left(x, f_{\varepsilon}\right)_{0}-\left(f_{\tilde{\varepsilon}}, f_{a, \varepsilon}\right)_{0}=$ $\left(f_{\varepsilon}, f_{a, \varepsilon}\right)_{0}=l_{S^{\prime}}$.

Soit $c$ une curvette associée à $S_{\varepsilon}$. Comme $n_{S^{\prime}}=1$, on a $:(x, \pi(c))_{0}=$ $\left(x, f_{\varepsilon}\right)_{0}$ et $\left(f_{\tilde{\varepsilon}}, \pi(c)\right)_{0}=\left(f_{\tilde{\varepsilon}}, f_{a, \varepsilon}\right)_{0}$. 
Le calcul de $\left(f_{\varepsilon}, f_{a, \varepsilon}\right)_{0}$ exprimé à l'aide de $l_{S_{\varepsilon}}$ montre que le nombre de sommets à ajouter est $m_{S^{\prime}}-m_{S_{\varepsilon}}=l_{S^{\prime}}-l_{S_{\varepsilon}} n_{S_{\varepsilon}}$.

Les points $\mathrm{c}$ des théorèmes 4 et 5 se démontrent comme suit.

Si $m_{\varepsilon}(f)>1$ et si $q_{S_{\varepsilon}}(f)<N$, alors on considère la géodésique d'une composante de $f_{a, \varepsilon}$ dans $\mathcal{A}\left(f \cdot f_{a} \cdot x\right)$. Elle contient exactement un sommet $S^{\prime}$ de quotient $N$, et les branches de $f_{a, \varepsilon}^{-1}(0)$ sont résolues en des curvettes de $S^{\prime}$. De plus, $\varepsilon$ est une arête de $\mathcal{A}\left(f \cdot f_{a} \cdot x\right)$ et toutes les géodésiques de $f_{\varepsilon}$ passent par $S^{\prime}$ dans $\mathcal{A}\left(f \cdot f_{a} \cdot x\right)$ (d'après la proposition 7 ).

On calcule la paire $\left(l_{S^{\prime}}, n_{S^{\prime}}\right)$ associée à $S^{\prime}$. Soit $c^{\prime}$ une curvette associée à $S^{\prime}$ et $c$ une curvette associée à $S_{\varepsilon}$. On obtient :

$$
N=\frac{\left(f, \pi\left(c^{\prime}\right)\right)_{0}}{\left(x, \pi\left(c^{\prime}\right)\right)_{0}}=\frac{\left(f_{a}, \pi\left(c^{\prime}\right)\right)_{0}}{\left(x, \pi\left(c^{\prime}\right)\right)_{0}} .
$$

Par conséquent on a : $N\left(x, \pi\left(c^{\prime}\right)\right)_{0}=\left(f_{a}, \pi\left(c^{\prime}\right)\right)_{0}=\left(f, \pi\left(c^{\prime}\right)\right)_{0}$, ou encore $N\left(x, \pi\left(c^{\prime}\right)\right)_{0}=\left(f_{\tilde{\varepsilon}}, \pi\left(c^{\prime}\right)\right)_{0}+\left(f_{\tilde{\varepsilon}}, \pi\left(c^{\prime}\right)\right)_{0}=\left(f_{a, \tilde{\varepsilon}}, \pi\left(c^{\prime}\right)\right)_{0}+\left(f_{a, \varepsilon}, \pi\left(c^{\prime}\right)\right)_{0}$.

Comme $\left(f_{a}, \pi(c)\right)_{0}=\left(f_{a, \varepsilon}, \pi(c)\right)_{0}+\left(f_{a, \tilde{\varepsilon}}, \pi(c)\right)_{0}$ et $(f, \pi(c))_{0}=\left(f_{\varepsilon}, \pi(c)\right)_{0}+$ $\left(f_{\tilde{\varepsilon}}, \pi(c)\right)_{0}$, par le corollaire 10 on a $\left(f_{a, \tilde{\varepsilon}}, \pi(c)\right)_{0}=\left(f_{\tilde{\varepsilon}}, \pi(c)\right)_{0}$.

Dans le cas I on obtient alors $N n_{S^{\prime}}(x, \pi(c))_{0}=n_{S^{\prime}}\left(f_{\tilde{\varepsilon}}, \pi(c)\right)_{0}+l_{S^{\prime}} m_{\varepsilon}(f)$, d'où $l_{S^{\prime}} / n_{S^{\prime}}=\omega$.

Dans le cas II nous avons $N \frac{n_{S^{\prime}}}{n_{S_{\varepsilon}}}(x, \pi(c))_{0}=\frac{n_{S^{\prime}}}{n_{S_{\varepsilon}}}\left(f_{\tilde{\varepsilon}}, \pi(c)\right)_{0}+l_{S^{\prime}} m_{\varepsilon}(f)$, d'où $l_{S^{\prime}} / n_{S^{\prime}}=\omega / n_{S_{\varepsilon}}$.

Ceci démontre le $\mathrm{c}$ du théorème 4 et la remarque 13 (ii) permet de conclure au c du théorème 5 .

\subsection{Cas dégénérés}

Les cas dégénérés concernent les valeurs de $N$ qui sont inférieures ou égales au plus petit quotient polaire de $f$. On trouve l'ensemble $B$ des valeurs atypiques du pinceau $f(x, y)+a x^{N}$ comme nous l'avons expliqué dans l'introduction. Ici on décrit le type topologique d'une fibre régulière en fonction du type topologique de $f \cdot x$.

THÉORÈme 6. - Pour a régulière, lorsque $N$ est inférieur ou égal au plus petit quotient polaire de $f$, alors le complémentaire dans $S_{\varepsilon}^{3}$ d'un voisinage tubulaire de l'entrelacs $f_{a}^{-1}(0) \cap S_{\varepsilon}^{3}$ est une variété de Seifert.

Ce théorème est une conséquence directe du lemme suivant : 
Lemme 3. - Pour a régulière, lorsque $N$ est inférieur ou égal au plus petit quotient polaire de $f$, on $a, Q_{a}=\{N\}$.

Démonstration. - La vérification de ce résultat est identique à la démonstration de la proposition 1 du paragraphe 2 .

Ce lemme peut aussi être obtenu à partir de résultats de B. Teissier ([T2]). Dans ce cas $f+a g^{N}=0$ apparaît comme l'intersection de $f+a z^{N}=0$ avec la surface $z=g(x, y)$.

Démonstration du théorème 6. - Le fait que $Q_{a}$ soit égal à $\{N\}$ se traduit par l'existence d'une unique zone polaire $Z_{1}$. Le lemme 2.5 .2 de [L-M-W1] nous dit alors que $\Sigma_{a} \cap \Phi_{a}^{-1}\left(Z_{1}\right)$ est connexe par arcs ; donc $\Sigma_{a}$ est connexe et par conséquent le complémentaire dans $\Sigma_{a}$ d'un voisinage tubulaire de l'entrelacs $\left(f_{a} \cdot x\right)^{-1}(0) \cap \Sigma_{a}$ est une variété de Seifert.

Corollaire 11. - Pour a régulière, l'arbre $A\left(f_{a} \cdot x\right)$ possède un unique sommet de rupture et les flèches qui représentent la transformée stricte de $f_{a}^{-1}(0)$ s'accrochent à ce sommet de rupture. En particulier $f_{a}$ a le type topologique de $x^{n}-y^{m}$ où $m=(f, x)_{0}$ et $n=\min \left(N,(f, y)_{0}\right)$.

Démonstration. - Nous venons de démontrer que le complémentaire dans $S_{\varepsilon}^{3}$ d'un voisinage tubulaire de l'entrelacs $\left(f_{a} \cdot x\right)^{-1}(0) \cap S_{\varepsilon}^{3}$ est une variété de Seifert. La correspondance biunivoque entre les variétés de Seifert de la décomposition minimale de Waldhausen du complémentaire dans $S_{\varepsilon}^{3}$ d'un voisinage tubulaire de l'entrelacs $\left(f_{a} \cdot x\right)^{-1}(0) \cap S_{\varepsilon}^{3}$ et les sommets de rupture de $A\left(f_{a} \cdot x\right)$ prouve la première partie du corollaire.

Soit $b$ une autre valeur régulière. L'image par $\Phi_{a}$ de $f_{b}^{-1}(0)$ est la courbe d'équation $v=(a-b) u^{N}$. C'est une feuille régulière. Par conséquent elle se relève par $\Phi_{a}$ en un nombre fini de feuilles régulières. Les composantes de $f_{b}^{-1}(0) \cap S_{\varepsilon}^{3}$ sont donc des feuilles régulières ; par conséquent leurs transformées strictes dans $\mathcal{A}\left(f_{a} \cdot x\right)$ sont symbolisées par des flèches du sommet de rupture. Comme $a$ et $b$ sont régulières, les types topologiques de $f_{a}$ et $f_{b}$ sont les mêmes, d'où le résultat.

\section{Exemples}

\subsection{Exemple 1}

Dans la suite la flèche blanche représente la transformée stricte de $\{x=$ $0\}$, les flèches noires les transformées strictes des composantes de $\{f=0\}$ et les flèches grises celles de $\left\{f_{a}=0\right\}$. 
Considérons $\Phi_{0}$ donné par $\Phi_{0}(x, y)=\left(x,\left(x^{5}-y^{3}\right)^{2}\right)$. Dans l'arbre $\mathcal{A}(f$. $x)$, chaque sommet $S_{i}$ est pondéré par le quotient de contact $q_{S_{i}}(f)=$ $\operatorname{val}_{E_{i}}(f) / \operatorname{val}_{E_{i}}(x)$ (voir figure 1).

On a $D(x, y)=-6 y^{2}\left(x^{5}-y^{3}\right) ; D^{-1}(0)$ possède donc deux branches. Pour $\left(x^{5}-y^{3}\right)$, dont une paramétrisation de Puiseux est donnée par $\left(t^{3}, t^{5}\right)$, on obtient $f\left(t^{3}, t^{5}\right)=0$; pour $\{y=0\}$, dont une paramétrisation de Puiseux est donnée par $(t, 0)$, on obtient $\operatorname{val}_{t}(f(t, 0))=10$ et $(x, y)_{0}=1$.

L'ensemble $Q_{0}$ est constitué du singleton $\{10\}$.

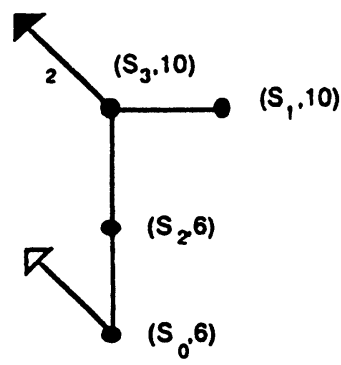

figure 1

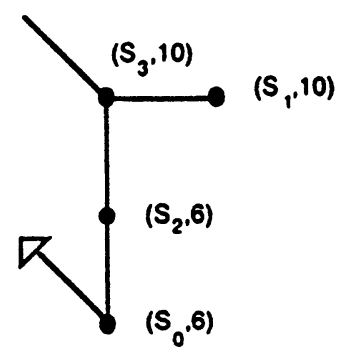

fieure 2

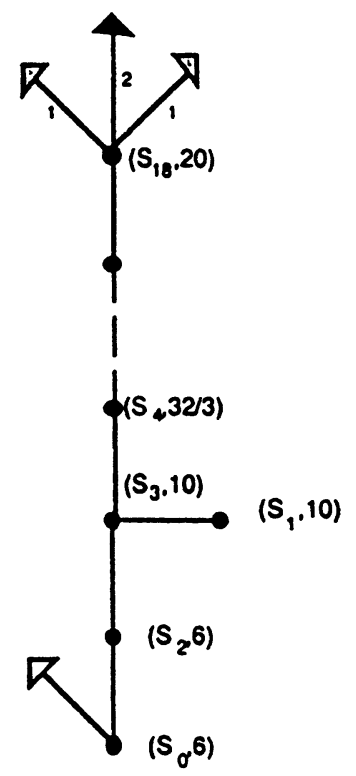

fixure 3

a) Pour $N=20$, l'arbre $\mathcal{A}_{20}(f \cdot x)$ est représenté figure 2 .

Dans ce cas $B_{1}=\emptyset$ et $B=\{0\}$ car $f$ n'est pas réduite.

Nous obtenons $Q_{a}=\{10 ; 20\}$ et en utilisant les théorèmes 4 et 5 nous trouvons que la zone de rupture créée est de paire $(30,1)$, ce qui nous permet de construire l'arbre $\mathcal{A}\left(f \cdot f_{a} \cdot x\right)$, où chaque sommet est pondéré par $q_{S_{i}}\left(f_{a}\right)=$ $\operatorname{val}_{E_{i}}\left(f_{a}\right) / \operatorname{val}_{E_{i}}(x)$ (voir figure 3).

b) Pour $N=10$ (voir l'arbre $\mathcal{A}_{10}(f \cdot x)$ figure 4), on a val $(f(t, 0))=$ $N(x, y)_{0}$. Par conséquent, $B_{1}=\{-1\}$ et $B=\{0,-1\}$ car $f$ n'est pas réduite. 
Si $a \in \mathbb{C} \backslash B, Q_{a}=\{10\}$ et la zone de rupture ajoutée est du type $(5,3)$, d'où la construction de $\mathcal{A}\left(f \cdot f_{a} \cdot x\right)$ (figure 5).

c) Pour $N=8, B_{1}=\emptyset$ et $B=\{0\}$ car $Q_{0}=\{10\}$. Par définition ce cas est dégénéré, et pour $a$ régulière l'arbre $\mathcal{A}\left(f_{a} \cdot x\right)$ est représenté figure 6 .

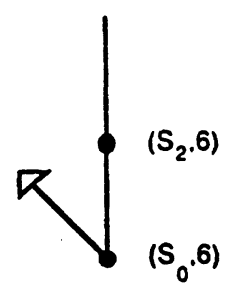

Fixure 4

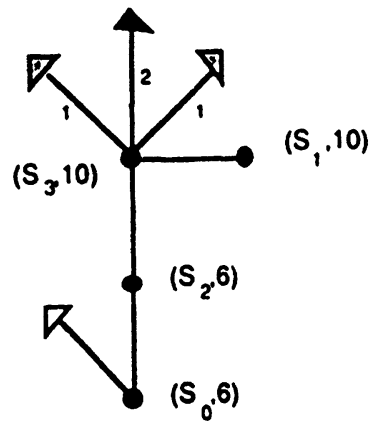

figure 5

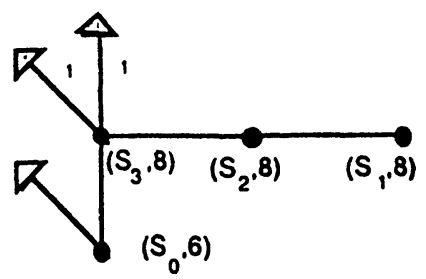

figure 6

\subsection{Exemple 2}

Considérons $f=\prod_{i=1}^{r} f_{i}^{e_{i}}$ où $r \geqslant 2$ et les $f_{i}$ sont lisses et transverses deux à deux. La multiplicité à l'origine de $f$ est donc égale à $\sum_{i=1}^{r} e_{i}$. On étudie $f(x, y)+a x^{N}$ avec $N>\sum_{i=1}^{r} e_{i}$. On a $q_{S_{1}}(f)=\sum_{i=1}^{r} e_{i}$ où $S_{1}$ est le sommet de $\mathcal{A}(f \cdot x)$ représentant le diviseur obtenu en éclatant l'origine dans $\mathbb{C}^{2}$. du pinceau $f+a x^{N}$.

LEMME 4 . Si $N>\sum_{i=1}^{r} e_{i}$ alors 0 est la seule valeur atypique éventuelle

Démonstration. - Si $x$ est transverse à $f$ alors $Q_{0}=\left\{\sum_{i=1}^{r} e_{i}\right\}$ et $N$ est strictement supérieur à l'unique quotient polaire de $f$ pour la direction $x$.

Dans le cas où $x$ n'est pas transverse à $f$, le quotient associé à $S_{0}$ est un quotient polaire de $f$ pour la direction $x$. Par le théorème de croissance, il est strictement inférieur à $\sum_{i=1}^{r} e_{i}$, donc à $N$. De plus, si $r \geqslant 3, \sum_{i=1}^{r} e_{i}$ est un deuxième quotient polaire pour $f$. Dans tous les cas $N$ est strictement supérieur aux quotients polaires de $f$ pour la direction $x$. 
La remarque 3 permet alors de conclure : si $f$ est réduite $B$ est vide, sinon $B=\{0\}$.

En appliquant le théorème 4 , a et $\mathrm{c}$, on construit $A\left(f_{a} \cdot x\right)$ à partir de $A(f$. $x$ ). Dans le cas c, on remplace chaque pointe de flèche correspondant à une composante irréductible $f_{i}$ de $f$ de poids $e_{i}>1$ par une zone possédant un unique sommet de rupture, de paire associée $\left(l_{(i)}, n_{(i)}\right)$, auquel on accroche $e_{i} / n_{(i)}$ flèches et pour lequel on a :

$$
l_{(i)} / n_{(i)}=\omega_{(i)}=\frac{N\left(x, f_{i}\right)_{0}-\sum_{j \neq i} e_{j}}{e_{i}} .
$$

\subsection{Exemple 3}

Comme dit dans l'introduction, notre caractérisation des valeurs atypiques d'un pinceau permet de déterminer les valeurs irrégulières à l'infini d'une application polynômiale de $\mathbb{C}^{2}$ dans $\mathbb{C}$ associée à un polynôme de $\mathbb{C}[x, y]$. Voici des exemples qui illustrent la méthode.

a) Supposons que $Q(x, y)$ est de la forme $\prod_{i=1}^{N}\left(x-a_{i}\right)$, les $a_{i}$ non nécessairement distincts. Les fibres de l'application polynômiale $Q$ sont des droites parallèles. Les valeurs irrégulières de $Q$ sont les valeurs $c \in \mathbb{C}$ telles que $Q(x)=c$ est non réduite. On les détermine de la façon suivante.

Soit $P(X, Z)=\prod_{i=1}^{N}\left(X-a_{i} Z\right)$ l'homogénéisée de $Q$. Le pinceau associé est $P(X, Z)+a Z^{N}=P_{a}(X, Z)$, et $P_{a}(X, Z) \cap\{Z=0\}=(0: 1: 0)=$ $\{A\}$. On localise au point $A$ et on calcule les valeurs atypiques du pinceau local $P(x, z)+a z^{N}$. Le germe $D$ défini dans l'introduction est le lieu des zéros de $\prod_{j=1}^{N-1}\left(x-c_{j} z\right)$, où les $c_{j}$ sont les racines de la dérivée du polynôme $\prod_{i=1}^{N}\left(x-a_{i}\right)$.

Quel que soit $j$ on a : $P\left(c_{j} z, z\right)=\left(\prod_{i=1}^{N}\left(c_{j}-a_{i}\right)\right) z^{N}$. On pose $b_{j}=$ $\prod_{i=1}^{N}\left(c_{j}-a_{i}\right)$. L'ensemble des valeurs atypiques de $Q$ est $B=\left\{-b_{j}, j=\right.$ $1, \ldots, N-1\}$ (les $b_{j}$ ne sont pas forcément distincts). 
b) En appliquant la même méthode qu'en a), on montre que $R(x, y)=$ $y^{q}+\prod_{i=1}^{N}\left(x-a_{i}\right)$ est régulier à l'infini si $q \geqslant 1$ et $q \neq N$.

c) Soit $S(x, y)=x+x^{\alpha} y^{\beta}$ avec $\alpha \geqslant 1$ et $\beta \geqslant 1$. Les fibres de $S$ intersectent la droite à l'infini en $A=(0: 1: 0)$ et $A^{\prime}=(1: 0: 0)$. Après localisation en $A$ (resp. $A^{\prime}$ ) on obtient les pinceaux : $x z^{\alpha+\beta-1}+x^{\alpha}+a z^{\alpha+\beta}$ (resp. $\left.z^{\alpha+\beta-1}+y^{\beta}+a z^{\alpha+\beta}\right)$.

En $A^{\prime}$ le germe $\hat{D}$ est $\beta y^{\beta-1}$ et alors $B=\emptyset$.

En $A$ le germe $\hat{D}$ est $z^{\alpha+\beta-1}+\alpha x^{\alpha-1}$. Les branches de $\hat{D}$ ont une paramétrisation de la forme $\left(z=t^{\alpha-1}, x=\sigma t^{\alpha+\beta-1}\right)$ avec $1+\alpha \sigma^{\alpha-1}=0$. Alors $B=\{0\}$. Donc la seule valeur atypique de $S$ à l'infini est 0 .

\section{Bibliographie}

[C1] CAUBEL (C.). - Sur La topologie d'une famille de pinceaux de germes d'hypersurfaces complexes, thèse de doctorat de l'université Paul Sabatier, Toulouse (1998).

[C2] CAUBel (C.). - Sur La topologie d'une famille de pinceaux de germes d'hypersurfaces complexes, C.R. Acad. Sci. Paris, t. 328, Série 1, (1999), 501504.

[C3] CAUBEL (C.). - Variation of the Milnor fibration in pencils of hypersurface singulartities, Proceedings of the London Math. Soc. Vol. 83, Part 2 (2001), 330-350.

[D-M] Du Bois (P.), Michel (F.). - The integral Seifert form does not determine the topology of plane curve germs, Journal of algebraic geometry, vol. $3, \mathrm{n}^{\circ} 1$, (1994),1-38.

[E-N] Eisenbud (D.), NeUMANN (W.). - Three-dimensional link theory and invariants of plane curves singularities, Annals of Math. Studies 110, (1985), Princeton University Press.

[I] IOMDINE (I.N.). - Complex surfaces with a 1-dimensional set of singularities, Sibirian Math. J. (15) (1974), 1061-1082.

[L1] LÊ (D.T.). - Calcul du nombre de cycles évanouissants d'une hypersurface complexe, Annales de l'Institut Fourier, t.23, $\mathrm{n}^{\circ} 4$, (1973), 261-270.

[L2] LÊ (D.T.). - Ensembles analytiques complexes avec lieu singulier de dimension un (d'après I.N. Iomdine), Séminaire sur les singularités, Université Paris VII, (1976-1977), Publications Mathématiques de l'université Paris VII, (1980).

[L-W] LÊ (D.T.), WeBER (C.). - Équisingularité dans les pinceaux de germes de courbes planes et $C^{0}$-suffisance, L'enseignement mathématique, t. 43 (1997), 355-380.

[L-M-W1] LÊ (D.T.), MiChel (F.), WeBER (C.). - Courbes polaires et topologie des courbes planes, Ann.Scien.E.N.S., 4ième série, T 24, (1991), 141-169. 
Fibrations associées à un pinceau de courbes planes

[L-M-W2] LÊ (D.T.), MiChel (F.), Weber (C.). - Sur le comportement des courbes polaires associées aux germes de courbes planes, Compositio Mathematica 72, (1989), 87-113.

[Ma1] MAUGendRE (H.). - Discriminant d'un germe $\Phi:\left(\mathbb{C}^{2}, 0\right) \longrightarrow\left(\mathbb{C}^{2}, 0\right)$ et résolution minimale de $f \cdot g$, Annales de la Faculté des Sciences de Toulouse, vol. VII, $\mathrm{n}^{0} 3$, (1998), 497-525.

[Ma2] MAUGendRe (H.). - Discriminant of a germ $\Phi:\left(\mathbb{C}^{2}, 0\right) \longrightarrow\left(\mathbb{C}^{2}, 0\right)$ and Seifert fibered manifolds, Journal of the London Mathematical Society (2) 59 (1999), 207-226.

[M-W] Michel (F.), WeBER (C.). - Topologie des germes de courbes planes à plusieurs branches, Prépublication de l'Université de Genève, (1985).

[Sc] SCHRAUWEN (R.). - Topological series of isolated plane curves singularities, l'Enseignement Mathématique (36) (1990), 115-141.

[S] SIERSMA (D.). - The monodromy of a series of hypersurface singumarities, Comment. Math. Helv. 65, (2), (1990), 181-197.

[T1] TeIssier (B.). - Introduction to equisingularity problems, Proc. A.M.S., Conference on algebraic geometry, Arcata 1974, A.M.S. Providence R.I. (1975).

[T2] TeIssien (B.). - Variétés polaires I, Inv. Math. 40 (1977), 267-292.

[Ti] TIBAR (M.). - Embedding non isolated singularities into isolated singularities, The Brieskorn Anniversary Volume, Birkhauser, Progress in Math., vol. 162, (1998), 103-115.

[Z] ZARISKI (O.). - Contributions to the problem of equisingularity, CIME notes (1969) ; Complete works vol.4, 159-237. 